\title{
Theoretical Investigation on Thick-walled Hub Structure Design of Subsea Connectors
}

\author{
Menglan Duan ${ }^{\text {a }}$, Kang Zhang ${ }^{\text {b* }}$, C. Guedes Soares ${ }^{c}$, Jeom Kee Paik ${ }^{\mathrm{d}, \mathrm{e}}$ \\ ${ }^{\text {a }}$ College of Safety and Ocean Engineering, China University of Petroleum, Beijing 102249, China \\ ${ }^{\mathrm{b}}$ China Ship Scientific Research Center, Wuxi 214082, China \\ ${ }^{c}$ Centre for Marine Technology and Ocean Engineering, Instituto Superior Técnico, Universdade \\ de Lisboa, Lisboa, 1049-001, Portugal \\ ${ }^{\mathrm{d}}$ Department of Naval Architecture and Ocean Engineering, Pusan National University, Busan \\ 46241, South Korea \\ e Department of Mechanical Engineering, University College London, London WC1E 7JE, UK
}

\begin{abstract}
Subsea processing of oil/gas is performed by production system facilities, which are linked into a complete system by subsea connectors. High reliability and long service life of the connectors, defined by strict requirement of deepwater oil fields, raise the challenge of the design of the structure components to which the ASME-VIII code has been applied. The theoretical design problems of the main parameters of subsea connector's hub structure are detailed in this paper in comparison with the shortage of the ASME design method. Therefore, a new analytical model is developed, which is called Stress Analytical Method (SAM). The hub structure is separated into two parts, i.e. a thick-walled cylinder and a hollow annular plate, and axisymmetric bending deformation problems of the thick-walled cylinder are proposed. The geometric equations, the constitutive equations, the physical equations and the equilibrium equations are developed to obtain the displacement analytical solution of the hub structure's thick-walled cylinder. The deformation continuity relationships between the thick-walled cylinder and the hollow annular plate are also established, and the analytical solutions of internal forces, displacements, rotation angles and stress components are acquired accordingly. The accuracy of SAM is investigated by comparing stress calculation results with Finite Element Method (FEM) results. A case study is carried out to compare SAM with ASME design method.
\end{abstract}

Key words: subsea production system; subsea connector; thick-walled cylinder; edge bending moment; edge shear force; axisymmetric bending deformation; stress;

\section{Introduction}

The increase of oil and gas production comes from offshore fields in the globe in the past decades, and about $90 \%$ of the increase is from the deepwater. Subsea production systems (SPS) including subsea pipelines and risers are the main equipment and facilities for the development of deepwater oil and gas fields, and their applications to deeper and remoter waters as well as arctic areas are challenging the scientists and engineers, especially in increasing safety and reliability. It is always the main concern of the industry to reduce the capital expenditures (CAPEX) and * Corresponding authors.

E-mail addresses: zhangkang5776@163.com (K. Zhang) 
operational expenditures (OPEX) of the deepwater fields by developing new technologies of subsea production systems. The technologies cover the whole period of life of the systems from initiation of concepts to design, manufacture, testing, transportation, installation, monitoring and inspection, maintenance, repair and decommissioning. Any technology shall aim for the maximum oil and gas production, the minimum environmental impact, the minimum risk to assets and personnel, the maximum profit and the highest safety of the subsea systems, and their advances are progressing each year.

A subsea production system consists of the seabed wellhead, subsea production X-tree, subsea tie-in to flowline system, and subsea equipment and control facilities to operate the well, as shown in Fig. 1. It can range in complexity from a single satellite well with a flowline linked to a fixed platform, FPSO (Floating Production, Storage and Offloading), or onshore facilities, to several wells on a template or clustered around a manifold that transfer to a fixed or floating facility or directly to onshore facilities. As the oil and gas fields move further offshore into deeper water and deeper geological formations in the quest for reserves, the technology of drilling and production has advanced dramatically. The latest subsea technologies have been proven and formed into an engineering system, namely, the subsea production system, which is associated with the overall process and all the equipment involved in drilling, field development, and field operation. More detailed information about subsea production system can be obtained in the report (Duan, et al. [1]).

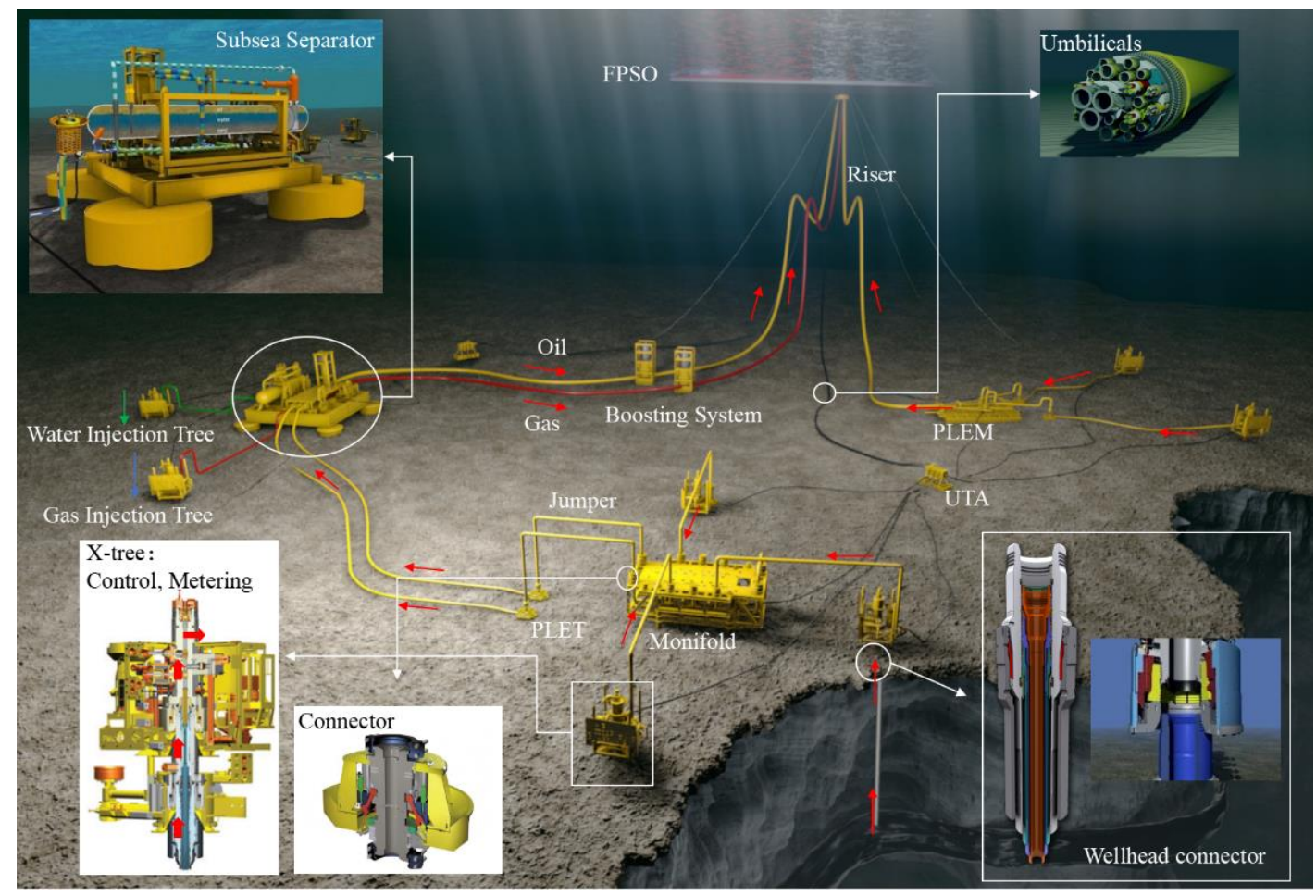

Fig. 1 Subsea production system (Duan, et al. [1])

The subsea connector, located on the end of the jumper, is the key connection facility in the subsea production system (shown in Fig.2). The main function of the subsea connector is to achieve locking and sealing between two different subsea facilities. If the sealing fails, oil and gas leakage accidents will occur. This will impact the safe operation of subsea production system. In the former study (Zhang et al., [2]), the sealing working principle of subsea connectors has been expressed, and the theoretical design method of the gasket, a key component of subsea connectors, has been developed. In the present work, the theoretical design method of the hub, another key component of 
subsea connectors, will be investigated. On the one hand, the hub component directly contacts with the gasket component and provides a compressive load on the gasket component to achieve sealing performance. On the other hand, the hub component contacts with the outer claw components, and cooperates with the claws to lock the locking mechanism. The design of hub component is constrained by the sealing performance. Meanwhile, the design of hub component will influence the locking performance of the subsea connector. Therefore, it will be of great significance to study the design methods of the structural parameters of the hub component. Among all the structural parameters of the hub component, the wall thickness dimension is the most significant. The reason is that the hub structure can be considered as the joint of two parts, i.e. a thick-walled cylinder and a flange ring plate, and the change of the two parts' wall thickness dimension seriously influences the global size of hub component. In order to ensure enough structural strength, the wall thickness dimension is designed very large in some cases, which will increase the global size of hub structure and make the whole structure of subsea connector much larger and heavier. Therefore, it is necessary to investigate the design methods for such key parameters of wall thickness of thick-walled cylinder and flange ring plate.

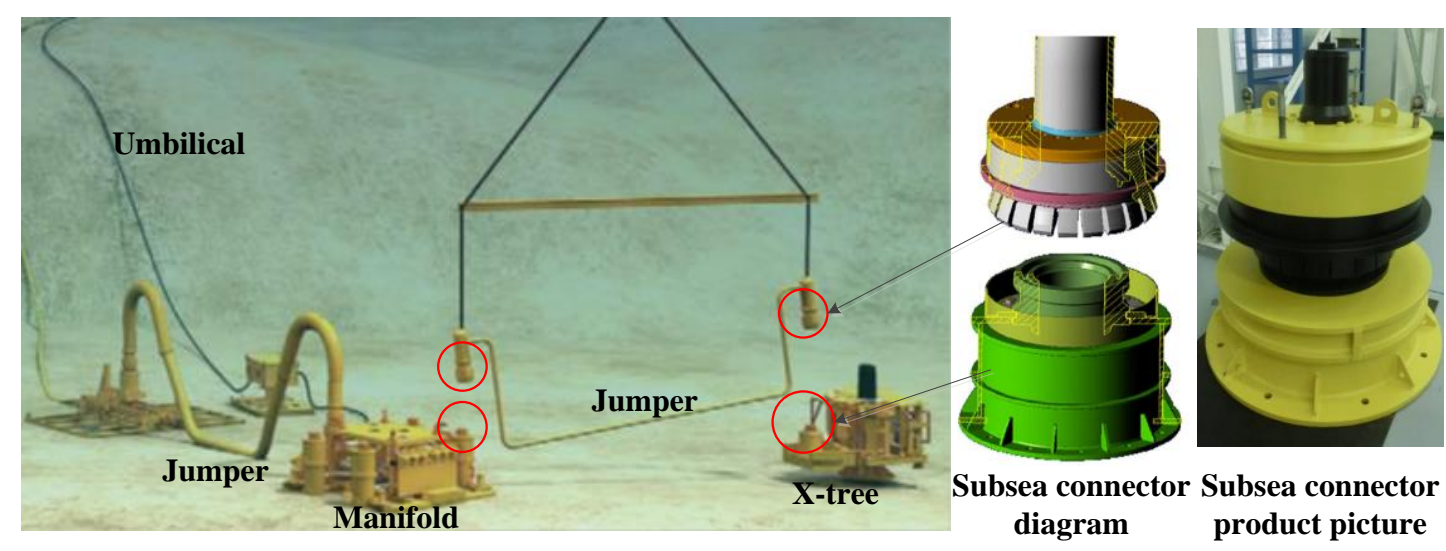

Fig. 2 Subsea production system and subsea connector

As a central part of the subsea connector, the typical locking system is composed of claws, an actuator ring, a gasket ring, upper hub and lower hub, as shown in Fig.3. It can be seen that the upper hub structure plays a key role in defining the geometrical dimensions of other components of the whole connector system, and the wall thickness of both the thick-walled cylinder and the flange ring plate shall be first set. Fig.4 presents the loading conditions of the hub structure respectively in locking and unlocking states. Zhang et al. [2] made a detailed presentation on the geometrical relationships and load transmission from the components in locking and unlocking conditions. Appendix A shows the calculation of such loads which are the basis of the derivation of the equations of this paper.

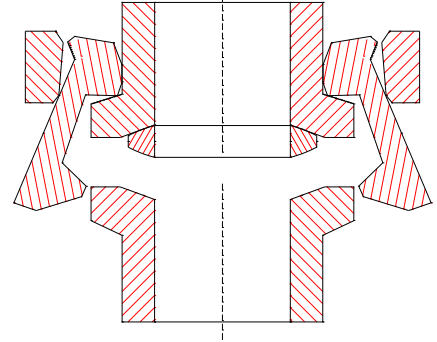

(a) unlocking state

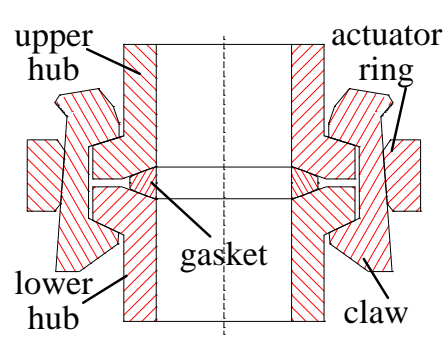

(b) locking state 
Fig. 3 A schematic presentation of the subsea connector locking system

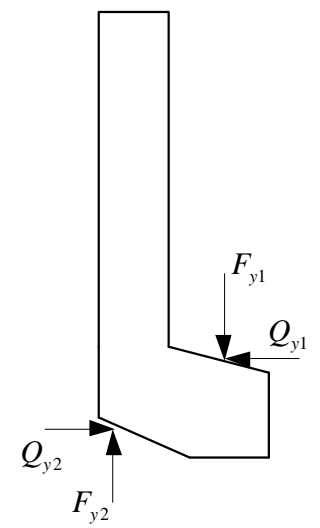

(a) Preloading state

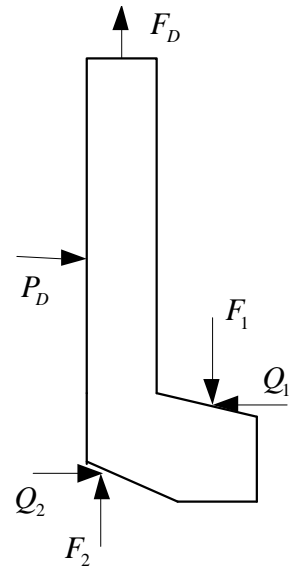

(b) Operation state

Fig. 4 Load conditions of the hub structure in two states

The ASME Boiler and Pressure Vessel VIII 2 [3] code is the general design method for the pressure vessel flange structure, where various sealing connection mechanisms have been elaborated, such as flat gasket sealing, double-cone gasket sealing, Uhde sealing, Casale sealing and clamp sealing. The design method is to calculate the stress components on critical sections and to make a judgement whether the design parameters meet the strength criteria or not. The hub structure of the subsea connector is similar to the flange of the clamp connection mechanism [4], and the terminal of the flange structure presents an outward expansion tendency under the combined actions of axial loads, axial moments and radial loads. As a result, an axisymmetric bending deformation takes place in the flange structure. The advantage of ASME design method is that the mechanics analytical model and the solution process are easy to be conducted. However, the biggest disadvantage is the approximations of the analytical model, which assumes the flange as a beam structure and neglects the continuous deformation consistent condition in the joint section between hub thick-walled cylinder and flange ring plate. Therefore, it is inevitable to cause errors when using the ASME design method to design the hub structure, and a more precise method should be developed to present the stress discontinuity.

The flange stress analysis method is the most widely used method to design the flange. In this method, the flange is separated into three parts: a hollow cylinder, the neck of the flange, and a ring [5]. The hollow cylinder, the neck of the flange and the ring are taken respectively as a thin-walled cylinder shell with constant thickness, a thin-walled cylinder shell with varying thickness, and a circular plate with a central hole. The axisymmetric bending equilibrium equations, the constitutive equations, the geometric equations and the boundary condition equations of the cylinder shell and the ring are established under the action of edge moment and the edge shear force. The usual approach is to assume the bending moment and the shear force as unknown variables and to utilize the two unknown variables to express the displacement and rotation angle equations of the cylinder shell edge, then to impose the compatibility conditions of deformations to solve the two unknown variables. Finally, analytical solutions of the displacement, rotation angle and stress components of the thin-walled cylinder shell edge are obtained, as can be seen in many literatures [6-14]. Such a stress analysis method not only takes into account discontinuous stress generated from the junction between cylinder and flange but also makes up for the drawbacks in ASME design method. 
However, the cylinder part of the subsea connectors' hub structure is comparatively large and thick, and a simple application of the stress analysis method of cone neck flange is limited. Therefore, the axisymmetric bending of the thick-walled cylinder should be investigated under the action of edge moment and edge shear force, rather than the axisymmetric bending of thin-walled cylinder shells. Some recent literatures about functionally graded rotating thick-walled hollow cylinder with variable thickness [15-18] also have to develop new models to obtain the analytical stress on the basis of thin wall-walled shell theory.

The space axisymmetric bending deformation theory is usually applied to the calculation of axisymmetric bending deformation of the thick-walled cylinder. The space axisymmetric bending deformation is one of classical elastic mechanics problems. The solution method of this deformation is generally finding a displacement function which is suitable to the bi-harmonic equation. Iyengar and Yogananda [19], Malova et al.[20], Vendhan and Archer [21], Ogaki and Nakajima [22], Chandrashekhara and Kumar [23], Li et al. [24], Ren [25], Zhu and Redekop [26], Chau and Wei [27], Meleshko and Tokovyy [28], and Wu et al. [29] have implemented such a method for solving practical engineering problems. The simplified models of these problems are all space axisymmetric bending deformations, and the axial stress boundary conditions of axial section is assumed to meet with some certain function distribution, and the shear stress is neglected. However, the axisymmetric bending deformation of the thick-walled cylinder of subsea connector is forced by the action of the edge load and edge shear force, and the shear force on the axial cross section will also take its effect. The above mentioned methods of solving such problems of axisymmetric bending deformation of thick-walled cylinder could not be simply applied when the shear force on the axial section could not be neglected.

To address the difficulties of the design of hub structural wall thickness in solving the problem of axisymmetric bending deformation of thick-walled cylinder under the action of the edge load and edge shear force, this paper will propose a new analytical model by critically analyzing the deformation theory of thin-walled cylinder shells. Taking the effect of the wall thickness into consideration, the geometric equations, the constitutive equations, the physical equations and the equilibrium equations are developed to obtain the displacement analytical solution of hub structure's thick-walled cylinder. Then the deformation continuity relationship between the thick-walled cylinder and the hollow annular plate is established, and the analytical solutions of internal forces, displacements, rotation angles and stress components are acquired accordingly. In this way, the analytical solution of the thick-walled cylinder axisymmetric bending deformation will be acquired under the action of edge loads. This analytical method is verified by FEM model, and a comparison investigation is made between the analytical method and ASME design method.

\section{Methodology}

\subsection{Overview on the ASME Design Method}

In the ASME design method, the hub structure is simplified into two parts, i.e. the thick-walled cylinder and the hollow annular plate (shown in Fig.5). Firstly, the internal loads, i.e. edge bending moment $M_{H}$ and edge shear force $Q_{H}$, on the joint section are calculated. Then the stress components are calculated by the internal loads, and finally the wall thickness parameters' design 
ranges are determined according to the strength criteria.

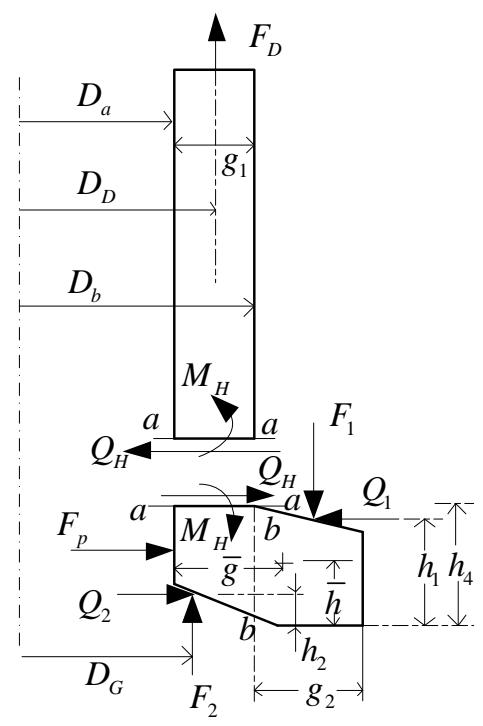

Fig. 5 Mechanics analysis on the hub structure

\section{(a) Solutions to Internal Loads}

The calculation methods of the edge moment $M_{H}$ and edge shear force $Q_{H}$ are expressed as:

$$
\begin{gathered}
M_{H}=\frac{M_{0}}{\varpi}=\frac{M_{0}}{\left\{1+\frac{1.818}{\sqrt{D_{a} g_{1}}}\left[h_{4}-\bar{h}+\frac{3.305 I_{h}}{g_{1}^{2}\left(0.5 D_{a}+\bar{g}\right)}\right]\right\}} \\
Q_{H}=1.818 \frac{M_{H}}{\sqrt{D_{a} g_{1}}}
\end{gathered}
$$

where, $\varpi$ is regarded as the correlation coefficient of total moment $M_{0}\left(M_{0}\right.$ is the total rotational moment on hub, and the calculation method can be seen in Appendix A.) and edge moment $M_{H} ; \varpi$, $\bar{g}$, and $I_{h}$ can be expressed as:

$$
\begin{gathered}
\varpi=\left\{1+\frac{1.818}{\sqrt{D_{a} g_{1}}}\left[h_{4}-\bar{h}+\frac{3.305 I_{h}}{g_{1}^{2}\left(0.5 D_{a}+\bar{g}\right)}\right]\right\} \\
\bar{g}=\frac{h_{4} g_{1}^{2}+h_{1} g_{2}\left(2 g_{1}+g_{2}\right)}{2\left(h_{4} g_{1}+h_{1} g_{2}\right)} \\
I_{h}=\frac{g_{1} h_{4}{ }^{3}}{3}+\frac{g_{2} h_{1}^{3}}{3}-\left(g_{1} h_{4}+g_{2} h_{1}\right) \bar{h}^{2}
\end{gathered}
$$

In the pre-loading state, the edge loads can be expressed as Eq.(6) and Eq.(7) by replacing $M_{0}$ by $M_{y 0}$ in Eq.(1) and Eq.(2).

$$
M_{y H}=\frac{M_{y 0}}{\left\{1+\frac{1.818}{\sqrt{D_{a} g_{1}}}\left[h_{4}-\bar{h}+\frac{3.305 I_{h}}{g_{1}^{2}\left(0.5 D_{a}+\bar{g}\right)}\right]\right\}}
$$




$$
Q_{y H}=1.818 \frac{M_{y H}}{\sqrt{D_{a} g_{1}}}
$$

\section{(b) Stress Components Calculation}

1) Section $a-a$

In operation state, the axial stress is superposed by the axial tension stress generated from the action of pressure on section $a-a$ and the bending stress caused by the edge bending moment $M_{H}$.

$$
\sigma_{o a}=\frac{D_{a}^{2}\left(P_{a}-P_{b}\right)}{4\left(D_{a}+g_{1}\right) g_{1}}+\frac{6 M_{H}}{\pi\left(D_{a}+g_{1}\right) g_{1}^{2}}
$$

In the pre-loading state, the axial stress is caused by the edge bending moment $M_{y H}$.

$$
\sigma_{\text {yoa }}=\frac{6 M_{y H}}{\pi\left(D_{a}+g_{1}\right) g_{1}^{2}}
$$

In the operation state, the hoop stress can be expressed by Lamé equations with the hub's cylinder being regarded as thick-walled cylinder. Therefore the maximum hoop stress can be expressed as:

$$
\sigma_{\theta \max }=\frac{\left(D_{b}^{2}+D_{a}^{2}\right) P_{a}-2 P_{b} D_{b}^{2}}{D_{b}^{2}-D_{a}^{2}}
$$

In the pre-loading state, the hoop stress is equal to 0 . In the operation state, the shear stress is caused by the edge shear force $Q_{H}$. The maximum shear stress is assumed as the 1.5 times of the average value in the calculation.

$$
\tau_{a}=1.5 \frac{Q_{H}}{\pi\left(D_{a}+g_{1}\right) g_{1}}
$$

In the pre-loading state, the shear stress can be expressed as:

$$
\tau_{y a}=1.5 \frac{Q_{y H}}{\pi\left(D_{a}+g_{1}\right) g_{1}}
$$

2) Section $b-b$

In the operation state, the shear stress is caused by the axial force $F_{1}$. The maximum shear stress is assumed as the 1.5 times of the average value in calculation.

$$
\tau_{b}=1.5 \frac{\pi D_{G} F_{2}+0.25 \pi D_{a}^{2}\left(p_{a}-p_{b}\right)}{\pi\left(D_{a}+2 g_{1}\right) h_{4}}
$$

where, the calculation method of $F_{2}$ can be seen in Appendix A.

In the pre-loading state, the shear stress can be expressed as:

$$
\tau_{y b}=1.5 \frac{\pi D_{G} F_{y 2}}{\pi\left(D_{a}+2 g_{1}\right) h_{4}}
$$

where, the calculation method of $F_{y 2}$ can be seen in Appendix A.

\section{(c) Design Criteria}


The shear force should not exceed 0.6 times of the materials' yield stress. The axial stress should not exceed the materials' yield stress. The hoop stress should not exceed two thirds of the yield stress. The specific design criteria are shown in Table 1.

Table 1 Design criteria for various stresses of flange for hub structure

\begin{tabular}{lc}
\hline \multicolumn{1}{c}{ Stress components } & Design criteria \\
\hline axial stress $\sigma_{o a}$ and $\sigma_{y o a}$ on section $a-a$ & $\sigma_{s}$ \\
the maximum hoop stress $\sigma_{\theta \max }$ on section $a-a$ & $\sigma_{s} / 1.5$ \\
the maximum shear stress $\tau_{a}$ and $\tau_{y a}$ on section $a-a$ & $0.6 \sigma_{s}$ \\
the maximum shear stress $\tau_{b}$ and $\tau_{y b}$ on section $b-b$ & $0.6 \sigma_{s}$ \\
\hline
\end{tabular}

With the above strength design criteria, the value ranges of the key parameters $g_{1}$ and $g_{2}$ can be determined by combining equations ranging from Eq.(8) to Eq.(14).

\subsection{The New Model with Solutions: Stress Analytical Method (SAM)}

A stress calculation model is shown in Fig.6, and the hub structure is also simplified into two parts, i.e. the thick-walled cylinder and the hollow annular plate. Based on the axisymmetric bending deformation theory of thin-walled cylinder shell, the mechanics analysis models are established for the thick-walled cylinder and hollow annular plate under the action of axisymmetric edge bending moment and edge shear force. This simplified approach is similar to the calculation of stress distributes of pressure vessels with a cylindrical shell and a head shell [30-31]. Through the deformation continuity conditions, the analytical solutions to internal forces, displacements, rotation angles and stress components are acquired. This method is called stress analytical method (SAM). And the specific process of SAM will be presented as follows:

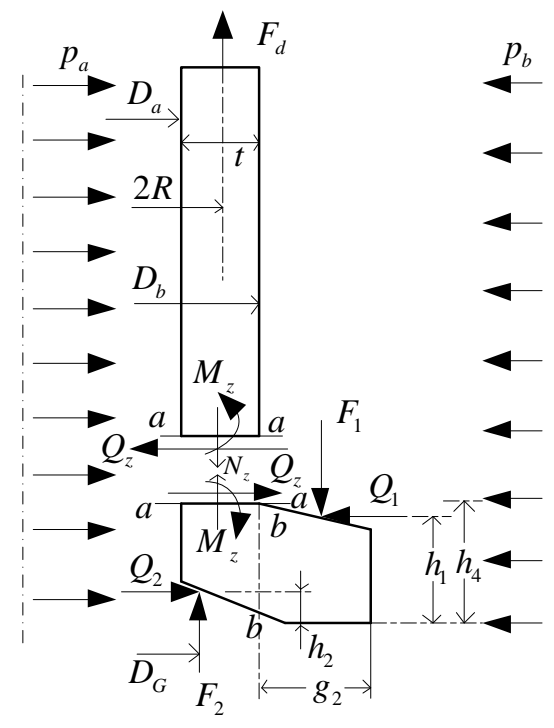

Fig. 6 Simplified diagram of mechanics analysis model of hub structure

Referring to the usual shell theory coordinates, the directions of $r$ and $z$ are shown in Fig.7. The directions of various forces and moments shown in Fig.8 are positive. 


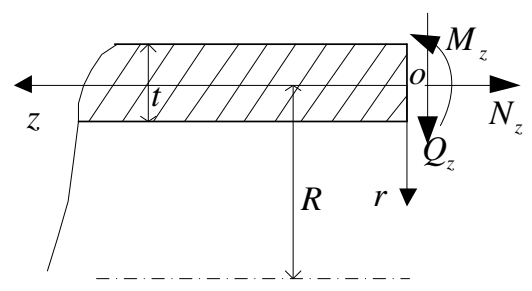

Fig. 7 Coordinates and cylinder geometry

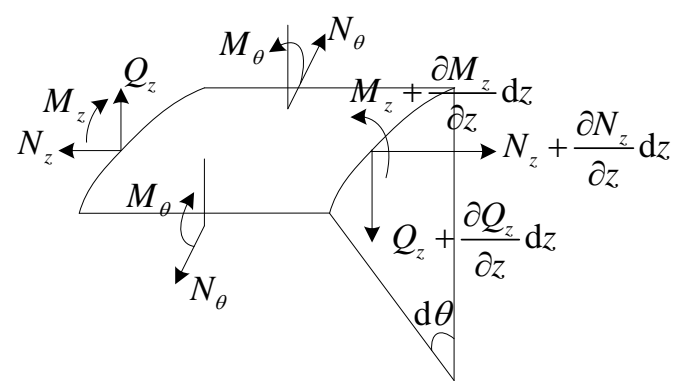

Fig. 8 Infinitesimal element of cylinder shell

\section{(1) Geometric Equations}

Considering the geometric equations of a thin-walled cylinder shell and the influence of wall thickness, the hoop strain $\varepsilon_{\theta}$ and the axial strain $\varepsilon_{z}$ can be expressed as:

$$
\left\{\begin{array}{l}
\varepsilon_{\theta}=-\frac{u_{r}}{R-r} \\
\varepsilon_{z}=\frac{d u_{z}}{d z}-r \frac{d^{2} u_{r}}{d z^{2}}
\end{array}\right.
$$

where, $R=\left(D_{b}+D_{a}\right) / 4, r$ is the radial coordinate, $r \in[-t / 2, t / 2]$. In the axisymmetric bending deformation theory of thin-walled cylinder shell [32-34], the expressions of $\varepsilon_{\theta}$ is without the symbol $r$, which means the influence of wall thickness is neglected.

\section{(2) Constitutive Equations}

Referring to Lamé theory solution of thick-walled cylinder, the radial stress can be expressed as:

$$
\sigma_{r}=A^{*} /(\mathrm{R}-r)^{2}+B^{*}
$$

Considering $\left.\sigma_{r}\right|_{r=t / 2}=-p_{a}$ and $\left.\sigma_{r}\right|_{r=-t / 2}=-p_{b}$, one can get $A^{*}=-\frac{\left(p_{a}-p_{b}\right) D_{a}^{2} D_{b}^{2}}{4\left(D_{b}^{2}-D_{a}^{2}\right)}$ and $B^{*}=\frac{D_{a}^{2} p_{a}-D_{b}^{2} p_{b}}{D_{b}^{2}-D_{a}^{2}}$.

The strain components can be expressed as:

$$
\left\{\begin{array}{l}
\varepsilon_{\theta}=\frac{1}{E}\left(\sigma_{\theta}-\mu \sigma_{z}\right)-\frac{\mu}{E} \sigma_{r} \\
\varepsilon_{z}=\frac{1}{E}\left(\sigma_{z}-\mu \sigma_{\theta}\right)-\frac{\mu}{E} \sigma_{r}
\end{array}\right.
$$

Combining Eq.(15) with Eq.(17) leads to the expressions of stress components by the displacement components :

$$
\left\{\begin{array}{l}
\sigma_{\theta}=\frac{E}{1-\mu^{2}}\left(-\frac{u_{r}}{R-r}+\mu \frac{d u_{z}}{d z}-\mu r \frac{d^{2} u_{r}}{d z^{2}}\right)+\frac{\mu}{1-\mu}\left[\frac{A^{*}}{(R-r)^{2}}+B^{*}\right] \\
\sigma_{z}=\frac{E}{1-\mu^{2}}\left(\frac{d u_{z}}{d z}-r \frac{d^{2} u_{r}}{d z^{2}}-\mu \frac{u_{r}}{R-r}\right)+\frac{\mu}{1-\mu}\left[\frac{A^{*}}{(R-r)^{2}}+B^{*}\right]
\end{array}\right.
$$

\section{(3) Physical Equations}


With the geometric equation and constitutive equation, the relation between internal loads and displacement components can be expressed as:

$$
\begin{gathered}
N_{\theta}=\int_{-t / 2}^{t / 2} \sigma_{\theta} d r=K\left(-\frac{u_{r}}{R}+\mu \frac{d u_{z}}{d z}\right)-\frac{D}{R} \frac{u_{r}}{R^{2}}+H_{1} \\
N_{z}=\int_{-t / 2}^{t / 2} \sigma_{z}\left(1-\frac{r}{R} d r=K \frac{d u_{z}}{d z}-\mu \frac{u_{r}}{R}+\right) \frac{D}{R} \frac{d^{2} u_{r}}{d z^{2}}+H_{2} \\
M_{z}=\int_{-t / 2}^{t / 2} \sigma_{z}\left(1-\frac{r}{R} \eta d r \Rightarrow D\left(\frac{d^{2} u_{r}}{d z^{2}} \frac{1}{R} \frac{d u_{z}}{d z}\right) H_{3}\right. \\
M_{\theta}=\int_{-t / 2}^{t / 2} \sigma_{\theta} r d r=D\left(-\mu \frac{d^{2} u_{r}}{d z^{2}}-\frac{u_{r}}{R^{2}}\right)+H_{4}
\end{gathered}
$$

where,

$H_{1}=\int_{-t / 2}^{t / 2} \frac{\mu}{1-\mu}\left[\frac{A^{*}}{(R-r)^{2}}+B^{*}\right] d r$,

$H_{2}=\int_{-t / 2}^{t / 2} \frac{\mu}{1-\mu}\left[\frac{A^{*}}{(R-r)^{2}}+B^{*}\right]\left(1-\frac{r}{R}\right) d r$,

$H_{3}=\int_{-t / 2}^{t / 2} \frac{\mu}{1-\mu}\left[\frac{A^{*}}{(R-r)^{2}}+B^{*}\right] r\left(1-\frac{r}{R}\right) d r$,

$H_{4}=\int_{-t / 2}^{t / 2} \frac{\mu}{1-\mu}\left[\frac{A^{*}}{(R-r)^{2}}+B^{*}\right] r d r$,

$D=\frac{E t^{3}}{12\left(1-\mu^{2}\right)}$,

$K=\frac{E t}{1-\mu^{2}}$. In the axisymmetric bending theory of thin-walled cylinder shell, $\sigma_{r}$ is equal to 0 , so $H_{1}, H_{2}, H_{3}$ and $H_{4}$ are omitted in constitutive equations.

\section{(4) Equilibrium Equations}

The equilibrium equations can be expressed as:

$$
\begin{gathered}
\frac{d N_{z}}{d z}=0 \\
\frac{d M_{z}}{d z}-Q_{z}=0 \\
R \frac{d Q_{z}}{d z}+N_{\theta}-\left[\left(R-\frac{t}{2}\right) p_{a}-\left(R+\frac{t}{2}\right) p_{b}\right]=0
\end{gathered}
$$

With three equilibrium equations and four physical equations, the equation set was developed for the axisymmetric bending deformation theory of thick-walled cylinder under the action of both internal and external pressures. In the equation set, there are seven unknown quantities and seven equations, so a unique solution can be obtained. By solving these equations, one obtains the displacement component, rotation angle and stress components.

According to Eq.(23), the axial force $N_{z}$ is constant. Assuming $N_{z}=N$, one can get $N=F_{d}=\frac{R}{2}\left(1-\frac{t}{2 R}\right)^{2}\left(p_{a}-p_{b}\right)$.

According to Eq.(20), one can get 
According to Eq.(19), one can get

$$
\frac{d u_{z}}{d z}=\frac{1}{K}\left(N-\frac{D}{R} \frac{d^{2} u_{r}}{d z^{2}}-H_{2}\right)+\mu \frac{u_{r}}{R}
$$

$$
N_{\theta}=K\left[\left(1+\frac{t^{2}}{12 R^{2}}\right) \frac{u_{r}}{R}+\mu \frac{d u_{z}}{d z}\right]+H_{1}
$$

Considering $\frac{t^{2}}{12 R^{2}} \ll 1$, Eq.(27) can be modified as:

$$
N_{\theta}=K\left[-\frac{u_{r}}{R}+\mu \frac{d u_{z}}{d z}\right]
$$

Substituting Eq.(26) into Eq.(28), one can get

$$
N_{\theta}=-\frac{K\left(1-\mu^{2}\right)}{R} u_{r}-\frac{\mu D}{R} \frac{d^{2} u_{r}}{d z^{2}}+\mu\left(N-H_{2}\right)+H_{1}
$$

Substituting Eq.(26) into Eq.(21), one can get

$$
M_{z}=D\left(-\frac{d^{2} u_{r}}{d z^{2}}-\frac{N-H_{2}}{R K}-\mu \frac{u_{r}}{R^{2}}\right)+H_{3}
$$

According to Eq.(24), Eq.(25), Eq.(29), and Eq.(30), one can get

$$
\frac{d^{4} u_{r}}{d z^{4}}+\frac{2 \mu}{R^{2}} \frac{d^{2} u_{r}}{d z^{2}}+\frac{\beta^{4}}{R^{4}} u_{r}=\frac{P}{D}
$$

where $\beta^{4}=\frac{12\left(1-\mu^{2}\right) R^{2}}{t^{2}}, P=-\left[\left(1-\frac{t}{2 R}\right) p_{a}-\left(1+\frac{t}{2 R}\right) p_{b}-\frac{\mu\left(N-H_{2}\right)+H_{1}}{R}\right]$.

By solving Eq.(31), the radial displacement component is expressed as:

$$
u_{r}=e^{-\lambda_{1} z}\left(A_{1} \cos \lambda_{2} z+A_{2} \sin \lambda_{2} z\right)+e^{\lambda_{1} z}\left(A_{3} \cos \lambda_{2} z+A_{4} \sin \lambda_{2} z\right)+\frac{P R^{4}}{D \beta^{4}}
$$

where,

$$
\begin{aligned}
& \lambda_{1}=\frac{\beta}{R} \sin \left(\frac{1}{2} \arccos \frac{\mu}{\beta^{2}}\right), \\
& \lambda_{2}=\frac{\beta}{R} \cos \left(\frac{1}{2} \arccos \frac{\mu}{\beta^{2}}\right) .
\end{aligned}
$$

The rotation angle is expressed as:

$$
\begin{aligned}
\theta=\frac{\partial u_{r}}{\partial z}= & -e^{-\lambda_{1} z}\left[\left(\lambda_{1} A_{1}-\lambda_{2} A_{2}\right) \cos \lambda_{2} z+\left(\lambda_{1} A_{2}+\lambda_{2} A_{1}\right) \sin \lambda_{2} z\right] \\
& +e^{\lambda_{1} z}\left[\left(\lambda_{1} A_{3}+\lambda_{2} A_{4}\right) \cos \lambda_{2} z+\left(\lambda_{1} A_{4}-\lambda_{2} A_{3}\right) \sin \lambda_{2} z\right]
\end{aligned}
$$

The stress components and the internal loads are expressed as follows :

$$
\begin{aligned}
& \sigma_{\theta}= \frac{\mu\left(N-H_{2}\right)}{t}+\frac{\mu^{2} R^{3} K}{\beta^{4} D t} P-\frac{R^{4} K}{\beta^{4} D t} P \frac{1}{R-r}+\frac{\mu}{1-\mu}\left[\frac{A^{*}}{(R-r)^{2}}+B^{*}\right] \\
&-\frac{K}{t} \frac{1}{R-r}\left[e^{-\lambda_{1} z}\left(A_{1} \cos \lambda_{2} z+A_{2} \sin \lambda_{2} z\right)+e^{\lambda_{1} z}\left(A_{3} \cos \lambda_{2} z+A_{4} \sin \lambda_{2} z\right)\right] \\
&--\frac{1}{t} e^{-\lambda_{1} z}\left\{(K r+D / R)\left[\mu\left(\lambda_{1}^{2}-\lambda_{2}^{2}\right)\left(A_{1} \cos \lambda_{2} z+A_{2} \sin \lambda_{2} z\right)+2 \mu \lambda_{1} \lambda_{2}\left(A_{1} \sin \lambda_{2} z-A_{2} \cos \lambda_{2} z\right)\right]\right. \\
&\left.-\left(\mu^{2} K / R\right)\left(A_{1} \cos \lambda_{2} z+A_{2} \sin \lambda_{2} z\right)\right\} \\
&-\frac{1}{t} e^{\lambda_{1} z}\left\{(K r+D / R)\left[\mu\left(\lambda_{1}^{2}-\lambda_{2}^{2}\right)\left(A_{3} \cos \lambda_{2} z+A_{4} \sin \lambda_{2} z\right)-2 \mu \lambda_{1} \lambda_{2}\left(A_{3} \sin \lambda_{2} z-A_{4} \cos \lambda_{2} z\right)\right]\right. \\
&\left.\quad-\left(\mu^{2} K / R\right)\left(A_{3} \cos \lambda_{2} z+A_{4} \sin \lambda_{2} z\right)\right\}
\end{aligned}
$$




$$
\begin{aligned}
& \sigma_{z}=\frac{\left(N-H_{2}\right)}{t}+\frac{\mu R^{3} K}{\beta^{4} D t} P-\frac{\mu R^{4} K}{\beta^{4} D t} P \frac{1}{R-r}+\frac{\mu}{1-\mu}\left[\frac{A^{*}}{(R-r)^{2}}+B^{*}\right] \\
& -\frac{\mu K}{t} \frac{1}{R-r}\left[e^{-\lambda_{1} z}\left(A_{1} \cos \lambda_{2} z+A_{2} \sin \lambda_{2} z\right)+e^{\lambda_{1} z}\left(A_{3} \cos \lambda_{2} z+A_{4} \sin \lambda_{2} z\right)\right] \\
& -\frac{1}{t} e^{-\lambda_{1} z}\left\{(K r+D / R)\left[\left(\lambda_{1}^{2}-\lambda_{2}^{2}\right)\left(A_{1} \cos \lambda_{2} z+A_{2} \sin \lambda_{2} z\right)+2 \lambda_{1} \lambda_{2}\left(A_{1} \sin \lambda_{2} z-A_{2} \cos \lambda_{2} z\right)\right]\right. \\
& \left.-(\mu K / R)\left(A_{1} \cos \lambda_{2} z+A_{2} \sin \lambda_{2} z\right)\right\} \\
& -\frac{1}{t} e^{\lambda_{1} z}\left\{(K r+D / R)\left[\left(\lambda_{1}^{2}-\lambda_{2}{ }^{2}\right)\left(A_{3} \cos \lambda_{2} z+A_{4} \sin \lambda_{2} z\right)-2 \lambda_{1} \lambda_{2}\left(A_{3} \sin \lambda_{2} z-A_{4} \cos \lambda_{2} z\right)\right]\right. \\
& \left.-(\mu K / R)\left(A_{3} \cos \lambda_{2} z+A_{4} \sin \lambda_{2} z\right)\right\} \\
& N_{\theta}=-\frac{\left(1-\mu^{2}\right) K R^{3}}{D \beta^{4}} P+\mu\left(N-H_{2}\right)+H_{1} \\
& +\frac{1}{R} e^{-\lambda_{1} z}\left\{\left[-K\left(1-\mu^{2}\right)-\mu D\left(\lambda_{1}^{2}-\lambda_{2}{ }^{2}\right)\right]\left(A_{1} \cos \lambda_{2} z+A_{2} \sin \lambda_{2} z\right)\right. \\
& \left.+2 \mu \lambda_{1} \lambda_{2} D\left(A_{1} \sin \lambda_{2} z-A_{2} \cos \lambda_{2} z\right)\right\} \\
& +\frac{1}{R} e^{\lambda_{1} z}\left\{\left[-K\left(1-\mu^{2}\right)-\mu D\left(\lambda_{1}^{2}-\lambda_{2}^{2}\right)\right]\left(A_{3} \cos \lambda_{2} z+A_{4} \sin \lambda_{2} z\right)\right. \\
& \left.-2 \mu \lambda_{1} \lambda_{2} D\left(A_{3} \sin \lambda_{2} z-A_{4} \cos \lambda_{2} z\right)\right\} \\
& Q_{z}=-D e^{-\lambda_{1} z}\left[-\lambda_{1}\left(\lambda_{1}^{2}-3 \lambda_{2}^{2}+\mu / R^{2}\right)\left(A_{1} \cos \lambda_{2} z+A_{2} \sin \lambda_{2} z\right)\right. \\
& \left.+\lambda_{2}\left(\lambda_{2}^{2}-3 \lambda_{1}^{2}-\mu / R^{2}\right)\left(A_{1} \sin \lambda_{2} z-A_{2} \cos \lambda_{2} z\right)\right] \\
& -D e^{\lambda_{1} z}\left[\lambda_{1}\left(\lambda_{1}^{2}-3 \lambda_{2}^{2}+\mu / R^{2}\right)\left(A_{3} \cos \lambda_{2} z+A_{4} \sin \lambda_{2} z\right)\right. \\
& \left.+\lambda_{2}\left(\lambda_{2}{ }^{2}-3 \lambda_{1}^{2}-\mu / R^{2}\right)\left(A_{3} \sin \lambda_{2} z-A_{4} \cos \lambda_{2} z\right)\right]
\end{aligned}
$$

Since the length of cylinder is much bigger than the radius, one can assume that the cylinder is semi-infinite length, so the radial displacement component can't increase to infinity. Then $A_{3}$ and $A_{4}$ should equal to 0 in Eq.(32). The Eq.(32) will be changed as:

$$
u_{r}=e^{-\lambda_{1} z}\left(A_{1} \cos \lambda_{2} z+A_{2} \sin \lambda_{2} z\right)+\frac{P R^{4}}{D \beta^{4}}
$$

The Eq.(39) will be changed as:

$$
\begin{aligned}
& M_{z}=-\frac{\mu R^{2}}{\beta^{4}} P-\frac{D\left(N-H_{2}\right)}{K R}+H_{3} \\
& -D e^{-\lambda_{1} z}\left[\left(\lambda_{1}^{2}-\lambda_{2}^{2}+\mu / R^{2}\right)\left(A_{1} \cos \lambda_{2} z+A_{2} \sin \lambda_{2} z\right)+2 \lambda_{1} \lambda_{2}\left(A_{1} \sin \lambda_{2} z-A_{2} \cos \lambda_{2} z\right)\right]
\end{aligned}
$$

The Eq.(37) will be changed as: 


$$
\begin{aligned}
Q_{z}=-D e^{-\lambda_{1} z} & -\lambda_{1}\left(\lambda_{1}^{2}-3 \lambda_{2}^{2}+\mu / R^{2}\right)\left(A_{1} \cos \lambda_{2} z+A_{2} \sin \lambda_{2} z\right) \\
+ & \left.\lambda_{2}\left(\lambda_{2}{ }^{2}-3 \lambda_{1}^{2}-\mu / R^{2}\right)\left(A_{1} \sin \lambda_{2} z-A_{2} \cos \lambda_{2} z\right)\right]
\end{aligned}
$$

Assuming $\left.M_{z}\right|_{z=0}=M_{e}$ and $\left.Q_{z}\right|_{z=0}==Q_{e}$, one can get expressions of the two coefficients $A_{1}$ and $A_{2}$ by the two unknown variables $M_{e}$ and $Q_{e}$.

$$
\begin{gathered}
A_{1}=\eta\left[\left(\frac{-M_{e}}{D}-\frac{N-H_{2}}{K R}-\frac{\mu R^{2}}{D \beta^{4}} P+\frac{H_{3}}{D}\right)\left(\lambda_{2}^{2}-3 \lambda_{1}^{2}-\mu / R^{2}\right)+2 \lambda_{1} \frac{Q_{e}}{D}\right] \\
A_{2}=\frac{\eta}{\lambda_{2}}\left[\left(\lambda_{1}^{2}-\lambda_{2}^{2}+\mu / R^{2}\right) \frac{Q_{e}}{D}-\lambda_{1}\left(\lambda_{1}^{2}-3 \lambda_{2}^{2}+\mu / R^{2}\right)\left(\frac{-M_{e}}{D}-\frac{N-H_{2}}{K R}-\frac{\mu R^{2}}{D \beta^{4}} P+\frac{H_{3}}{D}\right)\right]
\end{gathered}
$$

where, $\eta=-\frac{R^{4}}{R^{4}\left(\lambda_{1}^{2}+\lambda_{2}{ }^{2}\right)^{2}+2 \mu R^{2}\left(\lambda_{1}{ }^{2}-\lambda_{2}{ }^{2}\right)+\mu^{2}}$.

Then $u_{R}$ and $\theta_{R}$ on the section $z=0$ can be simplified as:

$$
\begin{gathered}
u_{R}=\frac{P R^{4}}{D \beta^{4}}+\eta\left[\left(\lambda_{2}^{2}-3 \lambda_{1}^{2}-\mu / R^{2}\right)\left(\frac{-M_{e}}{D}-\frac{N-H_{2}}{K R}-\frac{\mu R^{2}}{D \beta^{4}} P+\frac{H_{3}}{D}\right)+2 \lambda_{1} \frac{Q_{e}}{D}\right] \\
\theta_{R}=\eta\left[\left(\lambda_{1}^{2}+\lambda_{2}{ }^{2}-\mu / R^{2}\right) \frac{-Q_{e}}{D}+2 \lambda_{1}\left(\lambda_{1}^{2}+\lambda_{2}{ }^{2}\right)\left(\frac{-M_{e}}{D}-\frac{N-H_{2}}{K R}-\frac{\mu R^{2}}{D \beta^{4}} P+\frac{H_{3}}{D}\right)\right]
\end{gathered}
$$

With the continuity requirements of displacements and rotation angles, one can solve the unknown quantities $M_{e}$ and $Q_{e}$. The specific solving process is as follows:

On the section $z=0$, the radial displacement and rotation angle of hollow annular plate can be expressed as :

$$
\begin{gathered}
u_{R}^{*}=-\frac{12 M_{\text {total }} R^{2}}{E T^{2}\left(2 D_{c}-D_{a}\right)}=-\frac{12\left(M_{e}+0.5 Q_{e} T+M_{1}\right) R^{2}}{E T^{2}\left(2 D_{c}-D_{a}\right)} \\
\theta_{R}^{*}=-\frac{24 M_{\text {total }} R^{2}}{E T^{3}\left(2 D_{c}-D_{a}\right)}=-\frac{24\left(M_{e}+0.5 Q_{e} T+M_{1}\right) R^{2}}{E T^{3}\left(2 D_{c}-D_{a}\right)}
\end{gathered}
$$

where, $\quad M_{\text {total }}=M_{e}+0.5 Q_{e} T+M_{1}, \quad M_{1}=F_{1} H_{12}-Q_{1}\left(h_{1}-h_{2}\right)-F_{d} H_{d 2}, M_{1}$ is the bending moment generated from external loads. Here we assume that the radial displacement generated from $p_{a}, p_{b}$ and $Q_{e}$ are far less than the radial displacement caused by rotation, and just consider that the bending moment $M_{\text {total }}$ can affect the rotation angle and radial displacement.

According to the continuity requirements of displacements and rotation angles, the following equation set is obtained by combining the Eq. (39), Eq. (40), Eq. (41) and Eq. (42).

$$
\left\{\begin{array}{l}
u_{R}=u_{R}^{*} \\
\theta_{R}=\theta_{R}^{*}
\end{array}\right.
$$

In the equation set, there are two equations and two unknown quantities i.e. $M_{e}$ and $Q_{e}$, so a unique solution can be obtained. After getting the solutions to $M_{e}$ and $Q_{e}$, the analytical solutions to the internal force, the displacement, rotation angle and stress of the axisymmetric bending deformation of thick-walled cylinder under the effect of edge loads will be obtained by taking $M_{e}$ and $Q_{e}$ into equations ranging from Eq. (34) to Eq. (44).

After getting the analytical solutions of stress components, the strength checking criteria can be designated as Treca yield criteria or Mises yield criteria. Referring to the design criteria of ASME, the design criteria of SAM developed in this article are without adjustment for convenience 
to compare the designed parameters, as shown in Table 2.

Table 2 Design criteria for various stresses of flange for hub structure in SAM

\begin{tabular}{cc} 
Stress Type & Design Criteria \\
\hline the maximum axial stress $\sigma_{z}$ on the section $a-a$ & $\sigma_{s}$ \\
the maximum hoop stress $\sigma_{\theta}$ on the section $a-a$ & $\sigma_{s} / 1.5$ \\
the maximum shear stress $\tau_{a}=Q_{e} / g_{1}$ on the section $a-a$ & $0.6 \sigma_{s}$ \\
the maximum shear stress $\tau_{b}=1.5 F_{1} / h_{4}$ on the section $b-b$ & $0.6 \sigma_{s}$ \\
\hline
\end{tabular}

\section{Validation and Discussions}

To evaluate the accuracy of stress analytical solutions of SAM, finite element modeling analysis is firstly adopted at the present stage as thick shell elements or solid brick elements can easily be used to model thick shells in the commercial finite software. A 2D axisymmetric finite element model is developed by using structural parameters of one certain subsea connector. The axial stress and hoop stress are calculated for the thick-walled hub structure with loading the forces shown in Fig.4(b). In contrast, the axial stress and hoop stress are also calculated by SAM. The stresses of various points on several selected sections are assigned as comparison indexes.

\subsection{Calculation model}

The subsea connector's structural parameters (shown in Fig.9) are as follows:

Hub structure: $D_{a}=0.27 \mathrm{~m}, \mathrm{t}=\mathrm{g} 1=0.078 \mathrm{~m}, \mathrm{~g} 2=0.1 \mathrm{~m}, D_{G}=0.3 \mathrm{~m}, h_{1}=0.11 \mathrm{~m}, h_{2}=0.035 \mathrm{~m}$, $h_{4}=T=0.119 \mathrm{~m}, H=0.4 \mathrm{~m}, \alpha_{1}=20^{\circ}, \alpha_{3}=10^{\circ}$. Gasket structure: $R^{*}=0.4386 \mathrm{~m}, h=0.05 \mathrm{~m}$. $\delta=0.01 \mathrm{~mm}$.

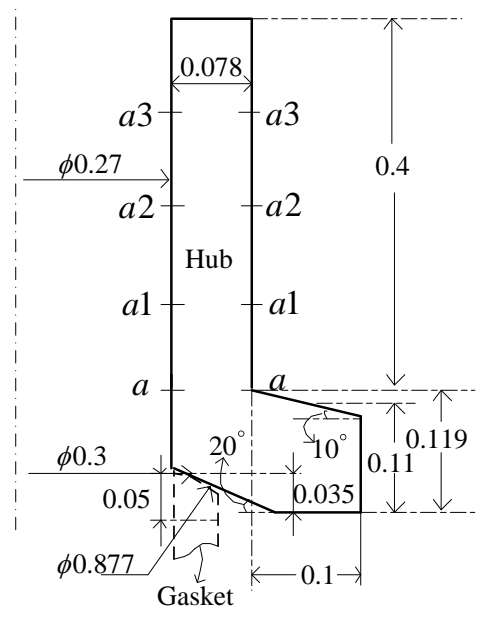

Fig.9 Subsea connector structural parameters

The material parameters are as follows: the elasticity modulus of hub structure is $210 \mathrm{GPa}$, the Poisson ratio of hub structure is 0.29 , the yield stress $\sigma_{s}$ of hub structure is $310 \mathrm{Mpa}$. The elasticity modulus of gasket ring structure is $205 \mathrm{GPa}$, and the Poisson ratio of gasket ring structure is 0.305 .

The loading parameters are as follows: the inner pressure $p_{\mathrm{a}}$ is $34.5 \mathrm{Mpa}$, and the outer pressure 
$p_{\mathrm{b}}$ is $15 \mathrm{Mpa}$.

Taking design parameters and material parameters of the gasket into Eq.(55)(shown in Appendix B), one can get $b=2.2 \mathrm{~mm}, p_{\max }=285.5 \mathrm{Mpa}, F_{b}=986.6 \mathrm{kN} / \mathrm{m}$. Then taking the three values into Eq.(52), Eq.(53), Eq. (58) and Eq.(59), the loads on each contact surface will be acquired. Assuming the friction force between contact faces is neglected, namely $\varphi_{1}=\varphi_{3}=0$, we can get $F_{1}=1635 \mathrm{kN} / \mathrm{m}, Q_{1}=288.3 \mathrm{kN} / \mathrm{m}, \quad F_{2}=613.8 \mathrm{kN} / \mathrm{m}, Q_{2}=223.4 \mathrm{kN} / \mathrm{m}, \quad F_{d}=1021.2 \mathrm{kN} / \mathrm{m}$. The five forces are loaded in SAM model for calculating the stress analytical solutions of thick-walled hub structure. The axial stress and hoop stress of eleven uniform-spaced points on four different sections, namely section $a-a(z=0)$, section $a 1-a 1(z=0.1 \mathrm{~m})$, section $a 2-a 2(z=0.2 \mathrm{~m})$, and section $a 3-a 3(z=0.3 \mathrm{~m})$, are concerned.

In the FEM model shown in Fig.10, the hub structure is assumed as an elastic body, while the gasket structure is assumed as a rigid body. The interaction condition is set as frictionless surface-to-surface contact for the hub and the gasket. The boundary conditions are that displacements in all directions are fixed on the gasket. The forces, $F_{1}=1635 \mathrm{kN} / \mathrm{m}, Q_{1}=288.3 \mathrm{kN} / \mathrm{m}$, and $F_{d}=1021.2 \mathrm{kN} / \mathrm{m}$, are loaded on corresponding locations and the pressure, $p_{a}$ and $p_{b}$, are loaded on corresponding surface. In this way, the reaction forces on hub structure produced by gasket structure, $F_{2}$ and $Q_{2}$, are ensured to be $613.8 \mathrm{kN} / \mathrm{m}$ and $223.4 \mathrm{kN} / \mathrm{m}$ respectively, which means $F_{2}$ and $Q_{2}$ are equivalently loaded on hub structure indirectly. The purpose of this approach is to guarantee that the loads in FEM model are the same as SAM model. Similarly, the axial stress and hoop stress of eleven uniform-spaced points on four different sections, namely section $a-a(z=0)$, section $a 1-a 1(z=0.1 \mathrm{~m})$, section $a 2-a 2(z=0.2 \mathrm{~m})$, and section $a 3-a 3(z=0.3 \mathrm{~m})$, are obtained and compared with those in SAM model. The consistency between SAM and FEM are investigated according to the comparison of stress values.

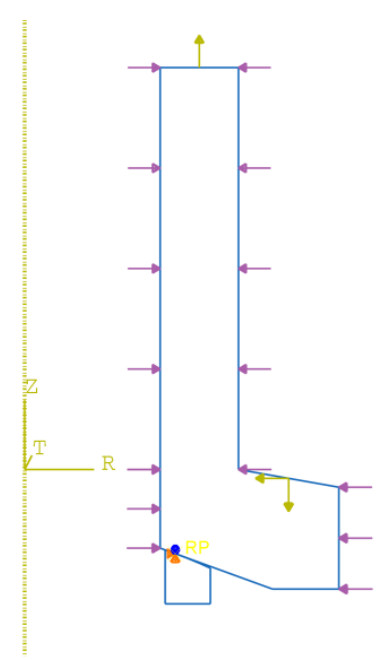

Fig.10 Loads and constrain conditions of FEM

In order to assess the adaptations to various wall thickness parameters for SAM model, changes of wall thickness parameter $t$ are made with the changeless of inner diameter $\mathrm{D}_{\mathrm{a}}$. Therefore, a new parameter $k$ is defined as the ratio of outer diameter $\mathrm{D}_{\mathrm{b}}$ and inner diameter $\mathrm{D}_{\mathrm{a}}$, namely $k=D_{b} / D_{a}=\left(D_{a}+2 t\right) / D_{a}$, which can represent the change of wall thickness $t$. The various values of $k$ are listed in Table 3. As the value $k$ is approximate 1.6 for the specific hub structural parameters shown in Fig.8, the value $k$ is regarded as 1.6, so the condition $k=1.6$ is not listed in Table 3. The load $F_{d}$ will change with the changes of wall thickness $t$, as well as $F_{1}$ and $Q_{1}$. Therefore, several groups of loads and wall thickness parameters under the various value conditions of $k$ are 
listed Table 3.

Table 3 Various $\boldsymbol{k}$ and corresponding wall thickness parameters and loads

\begin{tabular}{cccccc}
\hline$k$ & $T(\mathrm{~m})$ & $g_{2}(\mathrm{~m})$ & $F_{d}(\mathrm{kN} / \mathrm{m})$ & $F_{1}(\mathrm{kN} / \mathrm{m})$ & $Q_{1}(\mathrm{kN} / \mathrm{m})$ \\
\hline 1.3 & 0.04 & 0.138 & 1146.4 & 1760.2 & 310.4 \\
1.4 & 0.054 & 0.124 & 1096.9 & 1710.7 & 301.6 \\
1.5 & 0.068 & 0.11 & 1051.4 & 1665.2 & 293.6 \\
1.7 & 0.095 & 0.083 & 973.6 & 1587.4 & 279.9 \\
1.8 & 0.108 & 0.07 & 940.1 & 1553.9 & 274.0 \\
\hline
\end{tabular}

\subsection{Results discussion}

Axial stress and hoop stress of various points on several sections are compared under the conditions that $k=1.4, k=1.6$, and $k=1.8$, as shown in Fig.11, Fig.12, and Fig.13 respectively. The $x$-axis represents the different uniform-spaced points on section $z=0,0.1,0.2$, and 0.3 , where $r / t=-0.5$ represents the outer wall point, and $r / t=0.5$ represents the inner wall point.

The axial stress is regard as a comparison index to evaluate the consistency between SAM and FEM. As shown in Fig.11(a), the axial stress of outer wall point on section $z=0$ calculated by SAM has little difference with the one by FEM and the error is about $8 \mathrm{Mpa}$, while the axial stress of inner wall point on section $z=0$ calculated by SAM is distinguished from the one by FEM with a larger error value of $81 \mathrm{Mpa}$. Taking the stress value calculated by FEM as standard, the relative error of stress value of inner wall point by SAM is equal to $4 \%$ with a high consistency. The absolute value of axial stress of inner wall point calculated by SAM is close to that of the outer wall point with an error value of $20 \mathrm{Mpa}$, but the absolute value of axial stress of inner wall point calculated by FEM is far from the one of outer wall point with an error value of $110 \mathrm{Mpa}$. The reasons are that SAM is deduced on the basis of thin walled shell bending deformation theories, in which the radial displacement $u_{r}$ is assumed as one specific value on the whole section and is only correlation with $z$, the calculation formulas of axial stresses is originated from displacement solutions, and the main adaption of SAM is modifying geometric and constitutive equations to take the changes of curvature radius on different position $r$ into account, so the stress values calculated by SAM on inner wall point and outer wall point may be close. However, in the FEM model, the outer wall point on section $z=0$ is in the geometry discontinuity region, but the inner wall point is in the geometry continuity region, so the stress concentration phenomenon will occur on the outer wall point after loading external forces and the stress on outer wall point will inevitably exceed the inner wall point to a great extent. With the above illustrations, one can easily understand why the axial stress of outer wall point calculated by SAM and FEM are approximate but the axial stress of inner wall point shows a huge difference. On section $z=0$, the axial stress of outer wall point is a tension stress, but the axial stress of inner wall point is a compressive stress. The maximum stress of the whole section is on the outer wall point, so the outer wall point is the dangerous point on the section $z=0$. Although there is a large difference in the stress of inner wall point between FEM and SAM, the stress of outer wall point calculated by SAM is consistent with the one calculated by FEM. Fortunately, the outer wall point is the dangerous point of the whole section and the dangerous point is concerned once checking the structural strength, so the SAM model can provide helps to rapidly calculate the stress value of the dangerous point and quickly determinate rationalities of the design parameters. On the three sections $z=0.1, z=0.2$ and $z=0.3$, the stresses of 
outer wall point calculated by SAM and FEM are nearly close with a maximum error value of $3.8 \mathrm{Mpa}$, as well as the stresses of inner wall point. As the values of $\mathrm{z}$ increase, the calculation error of SAM will decrease. As shown in Fig.12(a) and Fig.13(a), similar regulars can be obtained that the error of the axial stress of inner wall point by SAM is large but the one of outer wall point is small, the stress value of the dangerous point calculated by SAM is consistent with the one calculated by FEM, and the calculation error of SAM will decrease once the section is away from $z=0$.

The hoop stress is also regarded as a comparison index to evaluate the consistency between SAM and FEM. As shown in Fig.12(b), the error of the hoop stress of the outer wall point on section $z=0$ calculated by SAM is about $13 \mathrm{Mpa}$ and the one of the inner wall point is about $17 \mathrm{Mpa}$. On section $z=0.1$, the error of the hoop stress of the outer wall point calculated by SAM is about $21 \mathrm{Mpa}$ and the one of the inner wall point is about $12 \mathrm{Mpa}$. On the two sections $\mathrm{z}=0.2$ and $\mathrm{z}=0.3$, the hoop stresses of outer wall point calculated by SAM and FEM are nearly close with a maximum error value of 10Mpa, as well as the stresses of inner wall point. In general, the error of the hoop stress calculated by SAM is acceptable. As the values of $z$ increase, the calculation error of the hoop stress calculated by SAM will decrease. As shown in Fig.13(b), similar regulars can be obtained that the hoop stresses of outer wall point calculated by SAM is consistent with the one calculated by FEM as well as the stresses of inner wall point. However, it can be seen from Fig.11(b) that the error of the hoop stress of the inner wall point on section $z=0$ calculated by SAM is about $26 \mathrm{Mpa}$ but the one of the outer wall point is relative large with a value of $45 \mathrm{Mpa}$. Apparently, on the condition of $k=1.4$, the error of the hoop stress of outer wall point by SAM is unacceptable in a certain degree, which reminds some limitations may exist in the SAM as for various wall thicknesses. Therefore, several groups of $k$, varying from 1.3 to 1.8 , are set and the corresponding calculation models are developed to investigate the possible limitations of SAM.

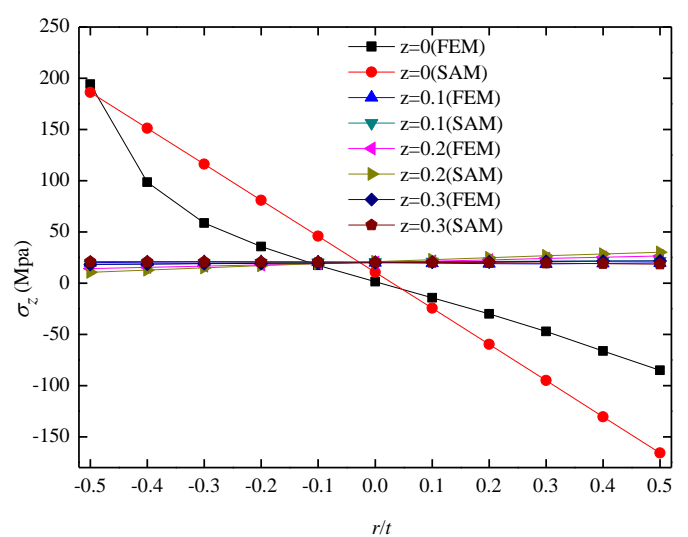

Fig.11(a) Comparison of axial stress on various sections when $k=1.4$

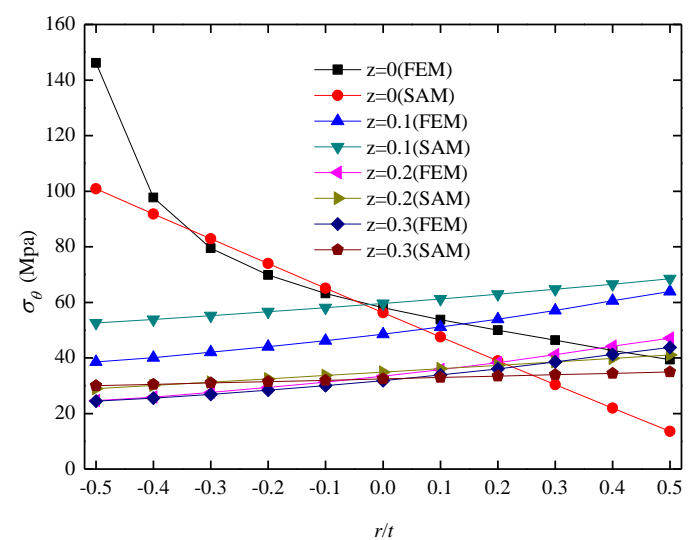

Fig.11(b) Comparison of hoop stress on various sections when $k=1.4$ 


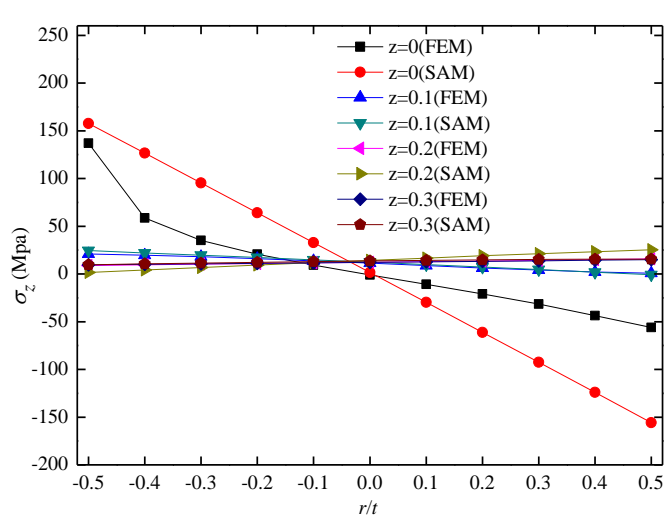

Fig.12(a) Comparison of axial stress on various sections when $k=1.6$

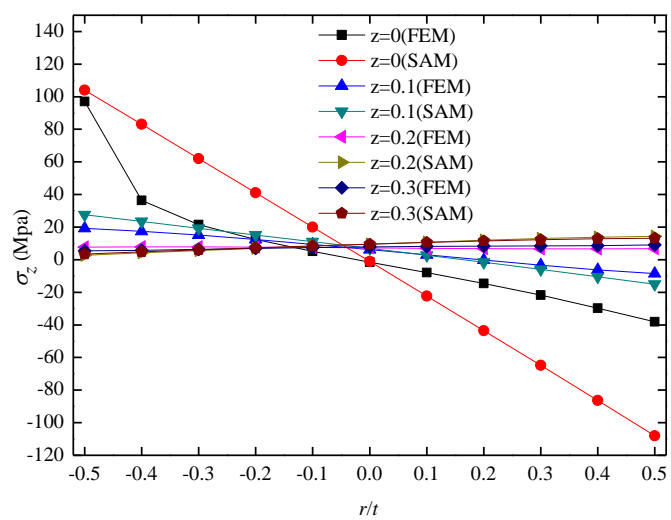

Fig.13(a) Comparison of axial stress on various sections when $k=1.8$

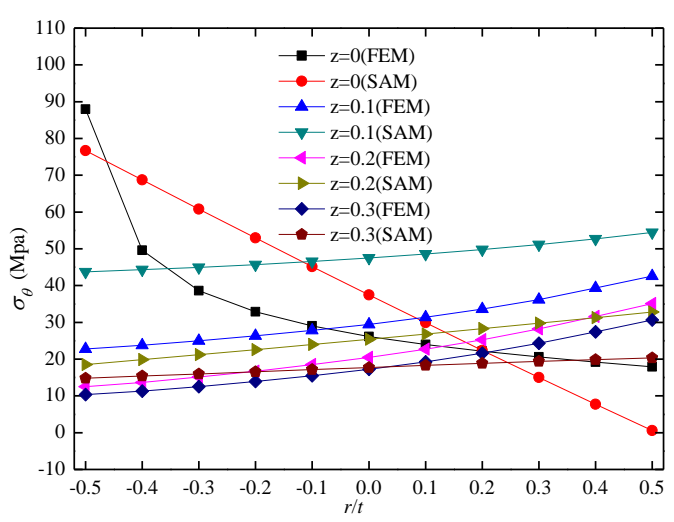

Fig.12(b) Comparison of hoop stress on various sections when $k=1.6$

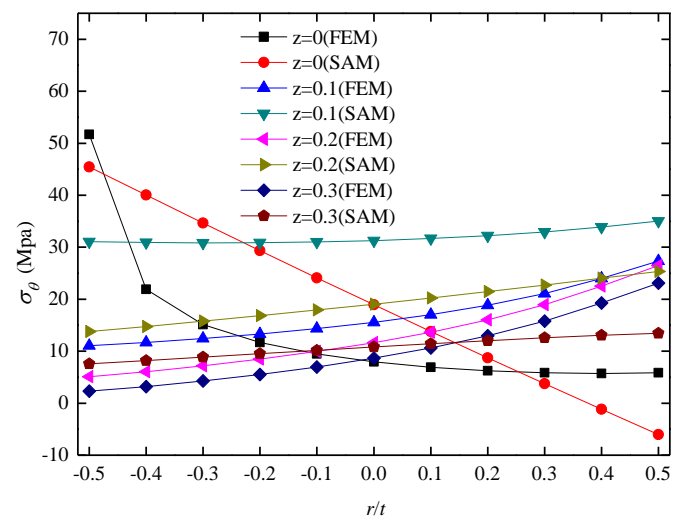

Fig.13(b) Comparison of hoop stress on various sections when $k=1.8$

As shown in Fig.14(a) and Fig.14(b), the axial stress of various points on section $z=0$ calculated by SAM is compared with the one calculated by FEM on different conditions of $k$. It can be seen that the error of the axial stress of the outer wall point calculated by SAM is about 66Mpa when $k$ is equal to 1.3. Taking the stress value calculated by FEM as standard, the relative error value of axial stress of outer wall point by SAM is equal to $28.6 \%$. However, the error of the axial stress of the outer wall point will fall down to $23 \mathrm{Mpa}$ and the relative error value is less than $20 \%$ once the value of $k$ is more than 1.4. Assuming the relative error value of $20 \%$ can be accepted in view of engineering problems, the axial stress values of the dangerous point calculated by SAM and FEM are consistent. Therefore, as for predicting the maximum axial stress, the recommended application limitation of SAM is $1.4 \leq k \leq 1.8$. As shown in Fig.15(a) and Fig.15(b), the hoop stress of various points on section $z=0$ calculated by SAM is compared with the one calculated by FEM on different conditions of $k$. It can be seen that the error of the hoop stress of the outer wall point calculated by SAM is about $94 \mathrm{Mpa}$ and the relative error value is equal to $48.6 \%$ when $k$ is equal to 1.3. On the condition of $k=1.4$, the error of the hoop stress of the outer wall point calculated by SAM is about $45.3 \mathrm{Mpa}$ and the relative error value is equal to $31 \%$. When the value of $k$ is more than 1.5, the error of the hoop stress of the outer wall point will fall down to $23 \mathrm{Mpa}$ and the relative error value is less than $18.1 \%$, which are acceptable. Therefore, as for predicting the maximum hoop stress, the recommended application limitation of SAM is $1.5 \leq k \leq 1.8$. Taking both the two comparison indexes of axial stress and hoop stress into consideration, the recommended application 
limitation of SAM is regard as $1.5 \leq k \leq 1.8$ with the calculation models developed in this paper and the acceptable relative error value of $20 \%$.

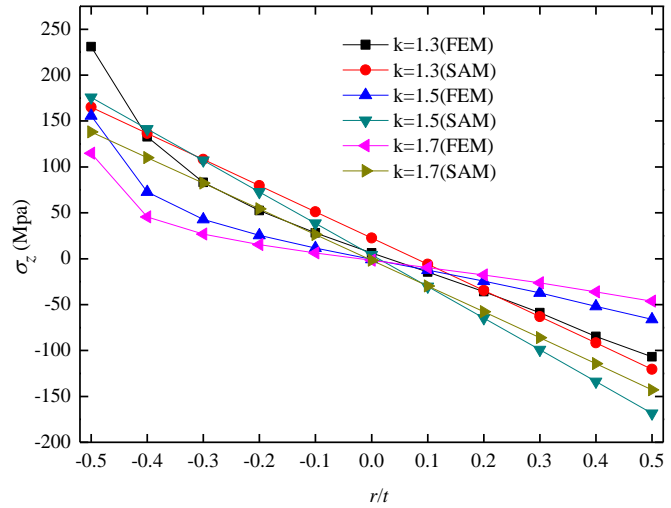

Fig.14(a). Comparison of axial stress on section $z=0$ when $k=1.3,1.5$ and 1.7

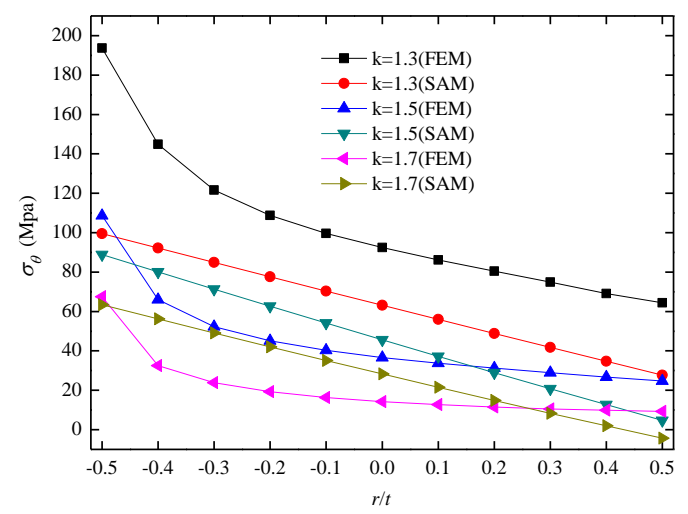

Fig.15(a). Comparison of hoop stress on section $z=0$ when $k=1.3,1.5$ and 1.7

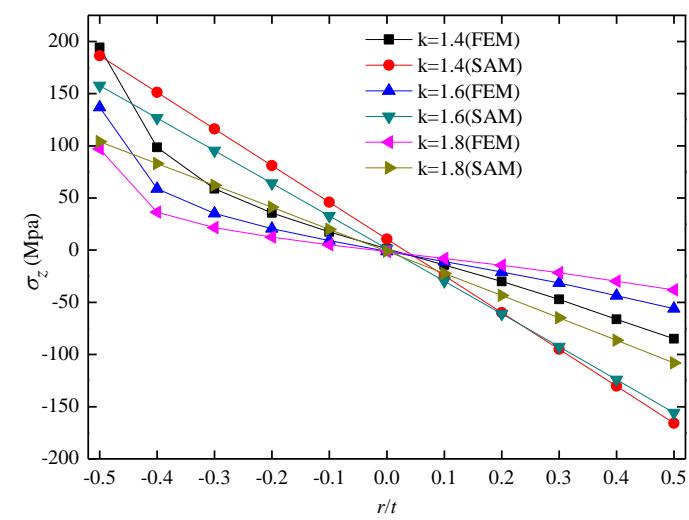

Fig.14(a). Comparison of axial stress on section $z=0$ when $k=1.4,1.6$ and 1.8

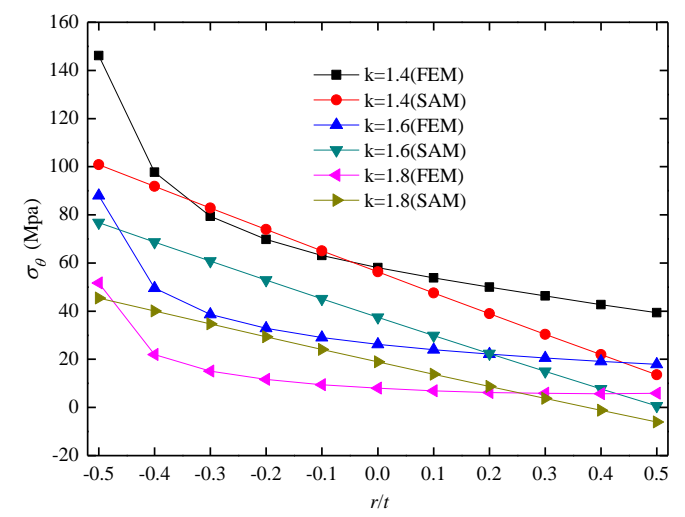

Fig.15(b). Comparison of hoop stress on section $z=0$ when $k=1.4,1.6$ and 1.8

\subsection{Design method comparison}

The axial stress data of the equally spaced points along the section $a-a$ are shown in Table 4 . Since the value of the axial stress or hoop stress obtained from ASME method is the maximum, the data in the first two columns are null value in Table 4. Taking the maximum axial stress value of 136.9 Mpa in FEM calculation results as a reference, the axial tension stress calculated by SAM is 157.7Mpa and the error value is $+20.8 \mathrm{Mpa}$, while the axial tension stress calculated by ASME method is $122.7 \mathrm{Mpa}$ and the error value is $-14.2 \mathrm{Mpa}$. The two error values are nearly consistent, and the advantages between SAM and ASME method cannot be distinguished easily by the error value. If the maximum axial tension stress value is adopted to check the structural strength for the wall thickness design parameters, SAM will be more conservative than ASME method, which will lead to designing a more reliable structure by SAM than by ASME method. What's more, taking the maximum hoop stress value of $88 \mathrm{Mpa}$ in FEM calculation results as a reference, the hoop stress calculated by SAM is $76.7 \mathrm{Mpa}$ and the error value is $-11.3 \mathrm{Mpa}$, while the axial hoop stress calculated by ASME method is $30.7 \mathrm{Mpa}$ and the error value is $-57.3 \mathrm{Mpa}$. Apparently, the error value in ASME method is larger than that in SAM. The advantage of SAM is more accurate in calculation of hoop stress than ASME method. Considering both the axial stress and hoop stress as comparison indexes, the security of the designed structure by SAM will be greatly improved. 
To sum up, the SAM not only can match well with the FEM calculation results but also make up the defects that ASME design method cannot accurately predict the hoop stress. Therefore SAM can be recommended as the design method for subsea connector's hub structure with a thick-walled cylinder. This method can also provide theoretical guidance for the design of subsea connectors.

Table 4 Contrast of stress on section $z=0$

\begin{tabular}{ccccccc}
\hline \multirow{2}{*}{$\begin{array}{c}\text { Point position } \\
\text { on the section }\end{array}$} & \multicolumn{2}{c}{$\begin{array}{c}\text { ASME design } \\
\text { method (Mpa) }\end{array}$} & \multicolumn{2}{c}{$\begin{array}{c}\text { SAM calculation results } \\
(\mathrm{Mpa})\end{array}$} & \multicolumn{2}{c}{$\begin{array}{r}\text { FEM calculation results } \\
\text { (Mpa) }\end{array}$} \\
\cline { 2 - 7 } & $\sigma_{z}$ & $\sigma_{\theta}$ & $\sigma_{z}$ & $\sigma_{\theta}$ & $\sigma_{z}$ & $\sigma_{\theta}$ \\
\hline $\begin{array}{c}0.5 \mathrm{t} \text { (inner wall } \\
\text { point) }\end{array}$ & - & - & -155.7 & 0.6 & -56.0 & 17.9 \\
$0.4 \mathrm{t}$ & - & - & -124.1 & 7.7 & -43.6 & 19.2 \\
$0.3 \mathrm{t}$ & - & - & -92.6 & 14.9 & -31.5 & 20.6 \\
$0.2 \mathrm{t}$ & - & - & -61.1 & 22.4 & -20.8 & 22.1 \\
$0.1 \mathrm{t}$ & - & - & -29.7 & 29.9 & -10.8 & 24.0 \\
0 & - & - & 1.6 & 37.5 & -1.0 & 26.2 \\
$-0.1 \mathrm{t}$ & - & - & 32.9 & 45.2 & 9.2 & 29.0 \\
$-0.2 \mathrm{t}$ & - & - & 64.1 & 52.9 & 20.7 & 32.8 \\
$-0.3 \mathrm{t}$ & - & - & 95.3 & 60.8 & 35.1 & 38.6 \\
$-0.4 \mathrm{t}$ & - & - & 126.5 & 68.7 & 58.7 & 49.6 \\
$-0.5 \mathrm{t}($ outer & 122.7 & 30.7 & 157.7 & 76.7 & 136.9 & 88.0 \\
wall point) & & & & & &
\end{tabular}

\section{Concluding remarks}

An analytical model of the hub structure's axisymmetric bending deformation is established in this paper, which can provide the solution to axisymmetric bending deformations of the thick-walled cylinder and the hollow annular plate under the action of edge bending moment and edge shear force. The displacement analytical solution to hub structure's thick-walled cylinder is obtained by the deformation continuity relationship between the thick-walled cylinder and the hollow annular plate. The analytical solutions of stress components are acquired accordingly. This analytical method is called Stress Analysis Method (SAM). Through comparing the analytical calculation results of axial stress and hoop stress with FEM results, the consistency of SAM is evaluated. A case study is carried out to compare the differences between SAM and the ASME design method. The conclusions are made as follows:

1) The stress analytical method developed in this paper solves the analytical calculation of the axisymmetric bending deformation and stress of thick-walled cylinder under the action of the edge bending moment and shear force, which can take into account the influence of thickness of the hub 
structure's thick-walled cylinder.

2) Considering both the axial stress and hoop stress as comparison indexes, the stress value of the dangerous point calculated by SAM is consistent with FEM results when the ratio of outer diameter and inner diameter of thick-walled cylinder is ranging from 1.5 to 1.8.

3) In comparison with the ASME design method, the stress analytical design method can get the more detail results and check out the weak points on the section by calculating the stresses along the whole section. The advantage of SAM is more accurate in predicting the hoop stress.

Through the SAM developed in this study, the analytical stress of the thick-walled hub structure will be obtained easily. The accuracy of SAM is validated by FEM model. In the future, the correlation experiment investigations are needed to improve the availability of SAM.

\section{Acknowledgements}

This work was financially supported by the National Key Research and Development Program of China (Grant No. 2016YFC0303702), the 111 Project of China (B18054), the Natural Science Foundation of Jiangsu Province of China (Grant No. BK20200165), the National Science and Technology Major Project of China (Grant No. 2016ZX05028-003-005).

\section{Appendix A Flange Ring's Total Bending Moment Calculation}

\section{Method}

$M_{0}$, an equivalent moment, is the total moment acting on hub's flange ring, which is applied in Eq.(1). The calculation method for $M_{0}$ is to summate the vector moments on the action line of $F_{1}$. $M_{0}$ can be expressed as:

$$
M_{0}=\pi D_{G} F_{2} H_{21}+F_{D} H_{D 1}+M_{p}-M_{R 2}-M_{R 1}
$$

where, $F_{2}$ is the reaction of gasket ring on hubs, the distance of action point between axial force $F_{1}$ and $F_{2}$ is expressed as $H_{21}=0.5\left(D_{b}-D_{G}+g_{2}\right) ; F_{D}$ is the equivalent axial force of pressure, and $F_{D}$ can be expressed as $F_{D}=\pi D_{a}^{2}\left(P_{a}-P_{b}\right) / 4$; The equivalent acting line of $F_{D}$ is on the middle section of the thick-walled cylinder. $H_{D 1}$ is the distance of action point between $F_{1}$ and $F_{D}$, which can be expressed as $H_{D 1}=0.5\left(g_{1}+g_{2}\right) ; M_{p}=\pi D_{a}\left(h_{4}-h_{2}\right)\left(P_{a}-P_{b}\right)\left[0.5\left(h_{4}-h_{2}\right)-\left(\bar{h}-h_{2}\right)\right]$, $M_{p}$ is the eccentric bending moment, because the equivalent force $F_{p}$ of $P_{a}$ and $P_{b}$ is not through the centroid. $\bar{h}=\frac{h_{4}^{2} g_{1}+h_{1}^{2} g_{2}}{2\left(h_{4} g_{1}+h_{1} g_{2}\right)}, \bar{h}$ is the axial distance between hub shoulder ring centroid and the surface of hub shoulder ring; $M_{R 2}=\pi D_{G} Q_{2}\left(\bar{h}-h_{2}\right), M_{R 2}$ is the moment generated from the force which is derived from the gasket ring in the horizontal direction; $M_{R 1}=\left(\pi D_{G} F_{2}+F_{D}\right) \tan \left(\alpha_{3}-\varphi_{3}\right)\left(h_{1}-\bar{h}\right), M_{R 1}$ is the moment generated from the force which is derived from the claw in the horizontal direction. The unit of all the moments is $\mathrm{N} \cdot \mathrm{m}$.

In the pre-loading state, $F_{D}$ and $M_{p}$ are equal to 0 in the Eq. (50), and the subscript of other forces' symbol will be added " $y$ " to differ from the operation state. Finally, the total moment $M_{y 0}$ can be expressed as:

$$
M_{y 0}=F_{y 2} H_{2}-M_{y R 2}-M_{y R 1}
$$


In the above equations, the derivation process of calculation equations of $F_{1}, Q_{1}, F_{2}$ and $Q_{2}$ are shown in the reference [2], and only the specific calculation equations are listed as follows:

(a) Sealing Loads $F_{2}$ and $Q_{2}$

The loads $F_{2}$ and $Q_{2}$ in the operation state are given in the reference [2] as follows:

$$
\begin{gathered}
F_{2}=\pi D_{G} \frac{F_{b}-0.5 \times 2 h \cdot\left(p_{a}-p_{b}\right) \sin \alpha_{1}}{\cos \varphi_{1}} \cos \left(\alpha_{1}+\varphi_{1}\right) \\
Q_{2}=F_{2} \tan \left(\alpha_{1}+\varphi_{1}\right)
\end{gathered}
$$

where, $F_{b}$ can be acquired from the contact model founded in in the reference[2], as shown in Eq.(54):

$$
\left\{\begin{array}{l}
\delta=\frac{E^{*} b^{2}\left(1-v_{1}^{2}\right)}{4 R^{*} E_{1}}\left(2 \ln \frac{2 h}{b \cos \alpha_{2}}-\frac{v_{1}}{1-v_{1}}\right) \\
p_{\max }=\frac{E^{*} b}{2 R^{*}} \\
F_{b}=\frac{\pi E^{*} b^{2}}{4 R^{*}}
\end{array}\right.
$$

The explicit expression of $b$ can be obtained by solving Eq. (54). Therefore the contact width, contact pressure and contact load can be directly calculated with the given design parameters, such as the compression amount $\delta$, the distance between top contact point and bottom contact point $2 h$, the radius of curvature $R^{*}$, and the slant angle of sealing contact surface $\alpha_{2}$.

$$
\left\{\begin{array}{l}
b=\sqrt{-2 m_{1} / \text { LambertW }\left(-1, \frac{-2 m_{1}}{\mathrm{~m}_{2}^{2}} \mathrm{e}^{2 \mathrm{~m}_{3}}\right)} \\
p_{\max }=\frac{E^{*} b}{2 R^{*}} \\
F_{b}=\frac{\pi E^{*} b^{2}}{4 R^{*}}
\end{array}\right.
$$

where, $m_{1}=\frac{2 R^{*} E_{1} \delta}{E^{*}\left(1-v_{1}^{2}\right)}, m_{2}=\frac{2 h}{\cos \alpha_{2}}, m_{3}=\frac{v_{1}}{2\left(1-v_{1}\right)}$, LambertW is a special function [35]. The Eq.(55) can be calculated directly in the MATLAB toolbox.

The loads $F_{y 2}$ and $Q_{y 2}$ under the pre-loading state are also given in the reference [2] as follows:

(b) Locking Loads $F_{1}$ and $Q_{1}$

$$
\begin{gathered}
F_{y 2}=\pi D_{G} \frac{F_{b}}{\cos \varphi_{1}} \cos \left(\alpha_{1}-\varphi_{1}\right) \\
Q_{y 2}=F_{y 2} \tan \left(\alpha_{1}-\varphi_{1}\right)
\end{gathered}
$$

The loads $F_{1}$ and $Q_{1}$ under the operation state are given in the reference [2] as follows :

$$
\begin{gathered}
F_{1}=F_{2}+0.5 R\left(1-0.5 t R^{-1}\right)^{2}\left(P_{a}-P_{b}\right) \\
Q_{1}=F_{1} \tan \left(\alpha_{3}-\varphi_{3}\right)
\end{gathered}
$$

Moreover, the loads $F_{y 1}$ and $Q_{y 1}$ under the pre-loading state are also given in the reference [2] as follows: 


$$
\begin{gathered}
F_{y 1}=F_{y 2} \\
Q_{y 1}=F_{y 1} \tan \left(\alpha_{3}+\varphi_{3}\right)
\end{gathered}
$$

\section{Nomenclature}

$D_{a} \quad$ Inner diameter of hub's thick-walled $D_{b}$ cylinder, $\mathrm{m}$

$D_{c} \quad$ External radius of hub's hollow annular plate, $\mathrm{m}$

$D_{G} \quad$ Diameter of the action position of $F_{2}, \mathrm{~m}$

$E_{1} \quad$ Elasticity modulus of gasket structure, $E^{*}$ pa

$F_{1} \quad$ Axial reaction force of the claw on hubs in operation state, $\mathrm{N} / \mathrm{m}$

$F_{b} \quad$ Contact force of gasket's sealing surface, $\mathrm{N} / \mathrm{m}$

$F_{\mathrm{y} 1} \quad$ Axial reaction force of the claw on hubs in preloading state, $\mathrm{N} / \mathrm{m}$

$F_{D} \quad$ Equivalent axial force of pressure, $\mathrm{N}$

$H_{12} \quad$ Radial distance of action point between axial force $F_{1}$ and $F_{2}, \mathrm{~m}$

$H_{d 2} \quad$ Radial distance of action point between $F_{2}$ and $F_{d}, \mathrm{~m}$

$I_{h} \quad$ Inertia of hub's hollow annular plate relative to its neutral axis, $\mathrm{m}^{4}$

$M_{1} \quad$ Bending moment generated from external loads of hub's hollow annular plate, $(\mathrm{N} \cdot \mathrm{m}) / \mathrm{m}$

$M_{p} \quad$ Eccentric bending moment, $\mathrm{N} \cdot \mathrm{m}$

$M_{y 0} \quad$ Total rotational moment on hub's hollow annular plate in preloading state, $\mathrm{N} \cdot \mathrm{m}$

$M_{y R 1}$ Eccentric Moment generated from the horizontal force derived from the claw in preloading state, $\mathrm{N} \cdot \mathrm{m}$

$M_{z} \quad$ Axial bending moment of the axial section of hub's thick-walled cylinder, $(\mathrm{N} \cdot \mathrm{m}) / \mathrm{m}$

$M_{R 1} \quad$ Eccentric Moment generated from the horizontal force derived from the claw in operation state, $\mathrm{N} \cdot \mathrm{m}$

$M_{\theta} \quad$ Circumferential moment of the axial section of hub's thick-walled cylinder, $(\mathrm{N} \cdot \mathrm{m}) / \mathrm{m}$

$N_{\theta} \quad$ Circumferential force of the axial section of hub's thick-walled cylinder, $\mathrm{N} / \mathrm{m}$

$Q_{1} \quad$ Radial reaction force of the claw on $Q_{2}$ hubs in operation state, $\mathrm{N} / \mathrm{m}$

$Q_{e} \quad$ Shear force of section $a-a, \mathrm{~N} / \mathrm{m}$
$D_{D}$

E

Outer diameter of hub's thick-walled cylinder , $\mathrm{m}$

Diameter of middle section of hub's thick-walled cylinder, $\mathrm{m}$

Elasticity modulus of hub structure, pa

Equivalent elasticity modulus of gasket and hub structure, pa

$F_{2} \quad$ Axial reaction force of the gasket ring on hubs in operation state, $\mathrm{N} / \mathrm{m}$

$F_{d} \quad$ Equivalent unit axial force of pressure , $\mathrm{N} / \mathrm{m}$

$F_{y 2} \quad$ Axial reaction force of the gasket ring on hubs in preloading state, $\mathrm{N} / \mathrm{m}$

$H$ Height of hub's thick-walled cylinder, $\mathrm{m}$

$H_{21} \quad$ Radial distance of action point between axial force $F_{1}$ and $F_{2}, H_{21}=H_{12}, \mathrm{~m}$

$H_{D 1} \quad$ Distance of action point between $F_{1}$ and $F_{D}, \mathrm{~m}$

$M_{0} \quad$ Total rotational moment on hub's hollow annular plate, $\mathrm{N} \cdot \mathrm{m}$

$M_{e} \quad$ Axial bending moment of section $a-a$, $(\mathrm{N} \cdot \mathrm{m}) / \mathrm{m}$

$M_{\text {total }}$ Total moment on hub's hollow annular plate, $(\mathrm{N} \cdot \mathrm{m}) / \mathrm{m}$

$M_{v H} \quad$ Edge bending moment in preloading state, $\mathrm{N} \cdot \mathrm{m}$

$M_{v R 2}$ Eccentric moment generated from the horizontal force derived from the gasket in preloading state, $\mathrm{N} \cdot \mathrm{m}$

$M_{H} \quad$ Edge bending moment in operation state, $\mathrm{N} \cdot \mathrm{m}$

$M_{R 2} \quad$ Eccentric moment generated from the horizontal force derived from the gasket in operation state, $\mathrm{N} \cdot \mathrm{m}$

$N_{z} \quad$ Axial force of the axial section of hub's thick-walled cylinder, $\mathrm{N} / \mathrm{m}$

$P_{D} \quad$ Equivalent radial force of pressure, $\mathrm{N}$

Radial reaction force of the gasket ring on hubs in operation state, $\mathrm{N} / \mathrm{m}$

$Q_{y 1} \quad$ Radial reaction force of the claw on hubs in preloading state, $\mathrm{N} / \mathrm{m}$ 
$Q_{y 2} \quad$ Radial reaction force of the gasket ring on hubs in preloading state, $\mathrm{N} / \mathrm{m}$

$Q_{H} \quad$ Edge shear force in operation state, $\mathrm{N}$

$R^{*} \quad$ Radius of curvature of the gasket's $T$ sealing surface, $\mathrm{m}$

$b \quad$ Half of contact width of gasket's sealing surface, $m$

$g_{2}$ Wall thickness of hub's hollow annular plate, $\mathrm{m}$

$h$

Half of the distance between top contact point and bottom contact point of gasket, $\mathrm{m}$

$h_{2} \quad$ Distance between the action position of $Q_{2}$ and the bottom edge of hub's hollow annular plate, $\mathrm{m}$

$\bar{h}$ Axial distance from the inner wall to the centroid of hub's hollow annular plate, $\mathrm{m}$

$p_{a} \quad$ Inner pressure, $\mathrm{pa}$

$p_{\max }$ The maximum contact pressure of $T$ gasket's sealing surface, pa

$u_{r} \quad$ Radial displacement , $m$

$u_{R} \quad$ Radial displacement of section $a-a$ on cylinder shell, $\mathrm{m}$

$\alpha_{1} \quad$ Inclination angle of the contact surface of gasket and hub structure, rad

$\sigma_{o a} \quad$ Axial stress of section a-a in operation state, pa

$\sigma_{s} \quad$ Yield stress of hub's material, pa

$\sigma_{z} \quad$ Axial stress, pa

$\sigma_{\theta \max }$ The maximum hoop stress of section a-a in operation state, pa

$\tau_{b} \quad$ Shear stress of section $b-b$ in operation state, pa

$\tau_{y b} \quad$ Shear stress of section $\mathrm{b}-\mathrm{b}$ in preloading state, pa

$\varepsilon_{\theta} \quad$ Hoop strain

$\varpi \quad$ Correlation coefficient of total moment $M_{0}$ and edge moment $M_{H}$

$\theta_{R} \quad$ Rotation angle of section $a$ - $a$ on cylinder shell, rad

$\varphi_{1} \quad$ Frictional angle of the contact surface of gasket and hub structure, rad

$v_{1} \quad$ Poisson ratio of gasket structure $p_{b}$

$Q_{z}$

$R$

$g_{1}$

$\bar{g}$

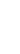

$h_{1}$

$k$

Ratio of outer diameter $\mathrm{D}_{\mathrm{b}}$ and inner diameter $\mathrm{D}_{\mathrm{a}}$

Outer pressure, pa

Wall thickness of hub's thick-walled cylinder, $t=g_{1}, \mathrm{~m}$

$u_{z} \quad$ Axial displacement, $\mathrm{m}$

$u_{R}^{*} \quad$ Radial displacement of section $a-a$ on hollow annular plate, $\mathrm{m}$

$\alpha_{3} \quad$ Inclination angle of the contact surface of claw and hub structure, rad

Radial stress, pa

Axial stress of section a-a in preloading state, pa

$\sigma_{\theta} \quad$ Hoop stress, pa

$\tau_{a}$

Shear stress of section a-a in operation state, pa

Shear stress of section a-a in preloading state, pa

Axial strain

Poisson ratio of hub structure

Rotation angle, rad

Rotation angle of section $a$ - $a$ on hollow annular plate, rad

Frictional angle of the contact surface of claw and hub structure, rad

Compression amount, $\mathrm{m}$

\section{References}

[1] Duan M L, Chai S H, Ilson P P, et al, . Committee V8: Report for Subsea Technology. Proceedings of the 20th International Ship and Offshore Structures Congress, Liege, Belgium. 
2018.

[2] Zhang K, Huang H, Duan M L, et al. Theoretical investigation of the compression limits of sealing structures in complex load transferring between subsea connector components. Journal of Natural Gas Science and Engineering. 44(2017)202-213.

[3]ASME Boiler and Pressure Vessel Code, VIII Division 2. Rules for Construction of Pressure Vessels. 2013.

[4]Dekker C J, Stikvoort W J. Improved design rules for pipe clamp connectors. International Journal of Pressure Vessels and Piping. 81(2)(2004)141-157.

[5]Cascales D H, Militello C. An accurate simple model to evaluate integral flange rotation. International Journal of Pressure Vessels \& Piping. 30(2)(1987)151-159.

[6] Magnucki K., Lewiński J. Fully stressed head of a pressure vessel. Thin-Walled Structures. 38(2000), 167-178.

[7] Zingoni A. Discontinuity effects at cone-cone axisymmetric shell junctions. Thin-Walled Structures. 40(2000) 877-891.

[8] Bouzid A H, Derenne M. Analytical modeling of the contact stress with nonlinear gaskets. Journal of pressure vessel technology. 124(2002)47-53.

[9] Galai H, Bouzid A H. Analytical modeling of flat face flanges with metal-to-metal contact beyond the bolt circle. Journal of pressure vessel technology. 132(2010), Article 061207.

[10] Zingoni A., Mokhothu B., Enoma N. A theoretical formulation for the stress analysis of multi-segmented spherical shells for high-volume liquid containment. Engineering Structures, 87(2015) 21-31.

[11] Yao T Y, He X H, Kong F S, et al. Design by analysis for orthotropic pressurized structure with small end of conical shell and cylindrical shell based on Hill48 yield criterion. Thin-Walled Structures. 96(2015)220-226.

[12] Pietraszkiewicz W., Konopinska V. Junctions in shell structures: A review. Thin-Walled Structures. 95(2015) 310-334.

[13] Eltyshev V., Chudinov P., Barykin Y. Analytical expressions for determining deflections, stresses and the required thickness of the orthotropic pressure cylinders. International Journal of Pressure Vessels and Piping. 175(2019) Article 103927.

[14] Zingoni A., Enoma N. Strength and stability of spherical-conical shell assemblies under external hydrostatic pressure, Thin-Walled Structures, 146(2020), Article 106472.

[15] Nejad M Z, Jabbari M, Ghannad M. Elastic analysis of axially functionally graded rotating thick cylinder with variable thickness under non-uniform arbitrarily pressure loading. International Journal of Engineering Science. 89(2015) 86-99.

[16] Benslimane A, Bouzidi S, Methia M. Displacement and stresses in pressurized thick-walled FGM cylinder: Exact and numerical solutions. International Journal of Pressure Vessel and Piping. 168(2018) 219-224.

[17] Khoshgoftar M J. Second order shear deformation theory for functionally graded axisymmetric thick shell with variable thickness under non-uniform pressure. Thin-Walled Structures. 144(2019) 86-99 Article 106286.

[18] Ghannad M, Rahimi G H, Nejad M Z. Elastic analysis of pressurized thick cylindrical shells with variable thickness made of functionally graded materials. Composites: Part B. 45(2013) 388-396.

[19]Iyengar K T, Yogananda C V. Long circular cylindrical laminated shells subjected to axisymmetric external loads. Journal of Applied Mathematics and Mechanics. 44(6)(1964)270-272. [20]Malova D G, Feldman E B, Ventskovskii B K. Investigation of the stress system in thick-walled cylinders under axisymmetrical axial load. Chemical \& Petroleum Engineering. 11(2)(1975)111-115.

[21]Vendhan C P, Archer R R. Axisymmetric stresses in transversely isotropic finite cylinders. International Journal of Solids \& Structures. 14(4)(1978)305-318.

[22] Ogaki Y, Nakajima N. Stress analysis of a circular cylinder of finite length subjected to loads 
symmetrical to middle plane and to axis of revolution. The science and engineering review of Doshisha University. 23(1983) 195-205.

[23]Chandrashekhara K, Kumar B S. Static analysis of a thick laminated circular cylindrical shell subjected to axisymmetric load. Composite Structures. 23(1)(1993)1-9.

[24]Li B L, Wang B R, Liu Z B, et al. Analysis solution of bulging convexity of the hydraulic elastic bulging roll. Chinese Journal of Mechanical Engineering (English Edition). 7(3)(1994) 198-204.

[25]Ren J G. Analysis of laminated circular cylindrical shells under axisymmetric loading. Composite Structures. 30(3)(1995)271-280.

[26] Zhu Y, Redekop D. Band loading of a thick-walled toroidal shell. International Journal of Pressure Vessel and Piping. 61(1995) 99-109.

[27]Chau K T, Wei X X. Finite solid circular cylinders subjected to arbitrary surface load. Part I-Analytic solution. International Journal of Solids and Structures. 37(40)(2000) 5707-5732.

[28]Meleshko V V, Tokovyy Y V. Equilibrium of an elastic finite cylinder under axisymmetric discontinuous normal loadings. Journal of Engineering Mathematics. 78(1)(2013) 143-166.

[29]Wu F, Wei X, Chen Z, et al. Numerical simulation and parametric analysis for designing High Energy Gas Fracturing. Journal of Natural Gas Science and Engineering. 53(2018) 218-236.

[30]Zingoni A , Enoma N , Govender N . Equatorial bending of an elliptic toroidal shell[J]. Thin-Walled Structures. 96(2015)286-294.

[31]Li C X, Yao T Y, He X H, et al. Stress analysis for the orthotropic pressurized structure of the cylindrical shell and spherical head[J]. Thin-Walled Structures. 111(2017)29-37.

[32] Flugge W. Stresses in Shells. Springer, Berlin, 1973

[33] Calladine C.R. Theory of Shell Structures. Cambridge University Press, Cambridge, 1983.

[34] Zingoni A. Shell Structures in Civil and Mechanical Engineering: Theory and Analysis. ICE Publishing, The Institution of Civil Engineers, London, 2018.

[35]Corless R M, Gonnet G H, Hare D, et al,. On the LambertW function. Advances in Computational Mathematics. 5(1)(1996) 329-359. 